\title{
New Improvement of the Expansion Methods for Solving the Generalized Fitzhugh-Nagumo Equation with Time-Dependent Coefficients
}

\author{
Jalil Manafian and Mehrdad Lakestani \\ Department of Applied Mathematics, Faculty of Mathematics Science, University of Tabriz, Tabriz 51569 84687, Iran \\ Correspondence should be addressed to Jalil Manafian; j_manafianheris@tabrizu.ac.ir
}

Received 30 May 2015; Revised 10 September 2015; Accepted 10 September 2015

Academic Editor: Josè A. Tenereiro Machado

Copyright (C) 2015 J. Manafian and M. Lakestani. This is an open access article distributed under the Creative Commons Attribution License, which permits unrestricted use, distribution, and reproduction in any medium, provided the original work is properly cited.

An improvement of the expansion methods, namely, the improved $\tan (\Phi(\xi) / 2)$-expansion method, for solving nonlinear secondorder partial differential equation, is proposed. The implementation of the new approach is demonstrated by solving the generalized Fitzhugh-Nagumo equation with time-dependent coefficients. As a result, many new and more general exact travelling wave solutions are obtained including periodic function solutions, soliton-like solutions, and trigonometric function solutions. The exact particular solutions contain four types: hyperbolic function solution, trigonometric function solution, exponential solution, and rational solution. We obtained further solutions comparing this method with other methods. The results demonstrate that the new $\tan (\Phi(\xi) / 2$ )-expansion method is more efficient than the Ansatz method and Tanh method applied by Triki and Wazwaz (2013). Recently, this method is developed for searching exact travelling wave solutions of nonlinear partial differential equations. Abundant exact travelling wave solutions including solitons, kink, and periodic and rational solutions have been found. These solutions might play an important role in engineering fields. It is shown that this method, with the help of symbolic computation, provides a straightforward and powerful mathematical tool for solving the nonlinear physics.

\section{Introduction}

Nonlinear evolution equations (NLEEs) are very important model equations in mathematical physics, engineering, and applied mathematics for describing diverse types of physical mechanisms of natural phenomena in the field of applied sciences, biochemistry, and engineering. Nonlinear wave equations play a major role in various fields such as plasma physics, fluid mechanics, optical fibers, solid state physics, chemical kinetics, geochemistry, and nonlinear optics $[1,2]$. Much work has been done over the years on the subject of obtaining the analytical solutions to the nonlinear partial differential equations (NPDEs). One of the most exciting advances of nonlinear science and theoretical physics has been the development of methods to look for exact solutions for NPDEs. The advent of symbolic computation also enables performing some complicated and tedious algebraic processes coupled with differential calculations. With the rapid development of nonlinear sciences based on computer algebraic system, many effective methods have been presented, such as the homotopy analysis method [3, 4], the variational iteration method [5-7], the homotopy perturbation method $[8,9]$, the sine-cosine method [10], the tanh-coth method [11-13], the modified extended tanhfunction method $[14,15]$, the Exp-function method [16, $17]$, the $\exp (-\Phi(\xi))$-expansion method $[18,19]$, the $\left(G^{\prime} / G\right)$ expansion method $[20,21]$, the modified simple equation method [22], the novel $\left(G^{\prime} / G\right)$-expansion method [23], the new approach of the generalized $\left(G^{\prime} / G\right)$-expansion method [21], the Jacobi elliptic function method [24], and the homogeneous balance method [25]. Recently, Naher and Abdullah [21] presented an effective and straightforward method, called the new approach of generalized $\left(G^{\prime} / G\right)$ expansion method, to obtain exact travelling wave solutions of nonlinear evolution equations. Here, we use an effective method, namely, the improved $\tan (\Phi(\xi) / 2)$-expansion 
method, for constructing a range of exact solutions for the following ordinary partial differential equations that in this paper we developed solutions as well. In this paper, we put forth the new approach of improved $\tan (\Phi(\xi) / 2)$-expansion method to construct exact travelling wave solutions including solitons, kink, and periodic and rational solutions to the generalized Fitzhugh-Nagumo equation with time-dependent coefficients. In $[22,26]$, the standard form of the FitzhughNagumo equation is given:

$$
u_{t}-u_{x x}+u(1-u)(\rho-u)=0
$$

where $0<\rho<1$ and $u(x, t)$ is of the unknown function depending on the temporal variable $t$ and the spatial variable $x$. When $\rho=-1$, (1) reduces to the real Newell-Whitehead equation which describes the dynamical behavior near the bifurcation point for the Rayleigh-Bénard convection of binary fluid mixtures [27]. The FN equation has various applications in the fields of flame propagation, logistic population growth, neurophysiology, branching Brownian motion process, autocatalytic chemical reaction, and nuclear reactor theory [28]. This equation combines diffusion and nonlinearity which is controlled by the term $u(1-u)(\rho-$ $u)$. Many physicists and mathematicians have paid much attention to the Fitzhugh-Nagumo equation in recent years due to its importance in mathematical physics. Remarkably, this nonlinear evolution equation is an important nonlinear reaction-diffusion equation and is usually used to model the transmission of nerve impulses [29-31]. Shih et al. utilized the approximate conditional symmetry method to determine approximate solutions admitted by a perturbation of (1). Equation (1) has been presented by Hariharan and Kannan via Haar wavelet method [32]. Kawahara and Tanaka [33] have found new exact solutions of (1), by applying the nonclassical symmetry reductions approach by using Hirota method. In [34], Nucci and Clarkson have obtained some new exact solutions with Jacobi elliptic function. Li and Guo [35] have obtained the new exact solutions of the Fitzhugh-Nagumo equation by using first integral method. In this paper, we consider that the generalized Fitzhugh-Nagumo equation with time-dependent coefficients is given as

$$
\begin{aligned}
u_{t}+\alpha(t) u_{x}-\beta(t) u_{x x} \\
\quad+\gamma(t)\left(\rho u-(1+\rho) u^{2}+u^{3}\right)=0,
\end{aligned}
$$

where $\alpha(t), \beta(t)$, and $\gamma(t)$ are arbitrary functions of $t$. The nonlinear models with variable coefficients are needed to describe the propagation of pulses. Triki and Wazwaz [36] obtained a new variety of soliton solutions by means of specific solitary wave Ansatz and the tanh method for (2). Also, Jiwari et al. [37] applied polynomial differential quadrature method to find the numerical solution of the generalized Fitzhugh-Nagumo equation with timedependent coefficients in one-dimensional space. In [38], the effect of diffusion on pattern formation in FitzHughNagumo model has been surveyed. Van Gorder applied the method of homotopy analysis to study the FitzhughNagumo equation [39]. In [40], a FitzHugh-Nagumo monodomain model has been used to describe the propagation of the electrical potential in heterogeneous cardiac tissue. Abbasian et al. have searched symmetric bursting behaviors in the generalized FitzHugh-Nagumo model [41]. Ray and Sahooz used the fractional subequation method for solving the space-time fractional Zakharov-Kuznetsov and modified Zakharov-Kuznetsov equations [42]. Also, the authors of [43] applied the fractional subequation method to the time fractional KdV-Zakharov-Kuznets and space-time fractional modified KdV-Zakharov-Kuznetsov equations. The purpose of this paper is to obtain exact solutions of the generalized Fitzhugh-Nagumo equation and to determine the accuracy of the improved $\tan (\Phi(\xi) / 2)$-expansion method in solving these kinds of problems. The paper is organized as follows. In Section 2, we describe the improved $\tan (\Phi(\xi) / 2)$-expansion method. In Section 3, we examine the generalized FitzhughNagumo equation with method introduced in Section 2. Also, conclusion and advantages are given in Section 4. Finally, some references are given at the end of this paper.

\section{Description of Improved $\tan (\Phi(\xi) / 2)$ - Expansion Technique}

Step 1. We suppose the given nonlinear fractional partial differential equation for $u(x, t)$ to be in the form

$$
\mathcal{N}\left(u, u_{x}, u_{t}, u_{x x}, u_{t t}, \ldots\right)=0
$$

which can be converted to an ODE:

$$
Q\left(u, u^{\prime},-\mu u^{\prime}, u^{\prime \prime}, \mu^{2} u^{\prime \prime}, \ldots\right)=0
$$

the transformation, $\xi=x-\mu t$, is wave variable. Also, $\mu$ is constant to be determined later.

Step 2. Suppose the traveling wave solution of (4) can be expressed as follows:

$$
\begin{aligned}
u(\xi)= & S(\Phi) \\
= & \sum_{k=0}^{m} A_{k}\left[p+\tan \left(\frac{\Phi(\xi)}{2}\right)\right]^{k} \\
& +\sum_{k=1}^{m} B_{k}\left[p+\tan \left(\frac{\Phi(\xi)}{2}\right)\right]^{-k}
\end{aligned}
$$

where $A_{k}(0 \leq k \leq m)$ and $B_{k}(1 \leq k \leq m)$ are constants to be determined, such that $A_{m} \neq 0, B_{m} \neq 0$, and $\Phi=\Phi(\xi)$ satisfies the following ordinary differential equation:

$$
\Phi^{\prime}(\xi)=a \sin (\Phi(\xi))+b \cos (\Phi(\xi))+c .
$$

We will consider the following special solutions of (6):

Family 1: When $a^{2}+b^{2}-c^{2}<0$ and $b-c \neq 0$, then $\Phi(\xi)=2 \arctan \left[a /(b-c)-\left(\sqrt{c^{2}-b^{2}-a^{2}} /(b-\right.\right.$ $\left.c)) \tan \left(\left(\sqrt{c^{2}-b^{2}-a^{2}} / 2\right)(\xi+C)\right)\right]$. 
Family 2: When $a^{2}+b^{2}-c^{2}>0$ and $b-c \neq 0$, then $\Phi(\xi)=2 \arctan \left[a /(b-c)+\left(\sqrt{b^{2}+a^{2}-c^{2}} /(b-\right.\right.$ $\left.c)) \tanh \left(\left(\sqrt{b^{2}+a^{2}-c^{2}} / 2\right)(\xi+C)\right)\right]$.

Family 3: When $a^{2}+b^{2}-c^{2}>0, b \neq 0$ and $c=0$, then $\Phi(\xi)=2 \arctan [a / b+$ $\left.\left(\sqrt{b^{2}+a^{2}} / b\right) \tanh \left(\left(\sqrt{b^{2}+a^{2}} / 2\right)(\xi+C)\right)\right]$.

Family 4: When $a^{2}+b^{2}-c^{2}<0, c \neq 0$ and $b=0$, then $\Phi(\xi)=2 \arctan [-a / c+$ $\left.\left(\sqrt{c^{2}-a^{2}} / c\right) \tan \left(\left(\sqrt{c^{2}-a^{2}} / 2\right)(\xi+C)\right)\right]$.

Family 5: When $a^{2}+b^{2}-c^{2}>0, b-$ $c \neq 0$ and $a=0$, then $\Phi(\xi)=$ $2 \arctan \left[\sqrt{(b+c) /(b-c)} \tanh \left(\left(\sqrt{b^{2}-c^{2}} / 2\right)(\xi+C)\right)\right]$.

Family 6: When $a=0$ and $c=0$, then $\Phi(\xi)=$ $\arctan \left[\left(e^{2 b(\xi+C)}-1\right) /\left(e^{2 b(\xi+C)}+1\right), 2 e^{b(\xi+C)} /\left(e^{2 b(\xi+C)}+\right.\right.$ 1)].

Family 7: When $b=0$ and $c=0$, then $\Phi(\xi)=$ $\arctan \left[2 e^{a(\xi+C)} /\left(e^{2 a(\xi+C)}+1\right),\left(e^{2 a(\xi+C)}-1\right) /\left(e^{2 a(\xi+C)}+\right.\right.$ 1)].

Family 8: When $a^{2}+b^{2}=c^{2}$, then $\Phi(\xi)=$ $-2 \arctan \left[((b+c)(a(\xi+C)+2)) / a^{2}(\xi+C)\right]$.

Family 9: When $a=b=c=k a$, then $\Phi(\xi)=$ $2 \arctan \left[e^{k a(\xi+C)}-1\right]$.

Family 10: When $a=c=k a$ and $b=-k a$, then $\Phi(\xi)=$ $-2 \arctan \left[e^{k a(\xi+C)} /\left(-1+e^{k a(\xi+C)}\right)\right]$.

Family 11: When $c=a$, then $\Phi(\xi)=-2 \arctan [((a+$ b) $\left.\left.e^{b(\xi+C)}-1\right) /\left((a-b) e^{b(\xi+C)}-1\right)\right]$.

Family 12: When $a=c$, then $\Phi(\xi)=2 \arctan [((b+$ c) $\left.\left.e^{b(\xi+C)}+1\right) /\left((b-c) e^{b(\xi+C)}-1\right)\right]$.

Family 13: When $c=-a$, then $\Phi(\xi)=$ $2 \arctan \left[\left(e^{b(\xi+C)}+b-a\right) /\left(e^{b(\xi+C)}-b-a\right)\right]$.

Family 14: When $b=-c$, then $\Phi(\xi)=$ $-2 \arctan \left[a e^{a(\xi+C)} /\left(c e^{a(\xi+C)}-1\right)\right]$.

Family 15: When $b=0$ and $a=c$, then $\Phi(\xi)=$ $-2 \arctan [(c(\xi+C)+2) / c(\xi+C)]$.

Family 16: When $a=0$ and $b=c$, then $\Phi(\xi)=$ $2 \arctan [c(\xi+C)]$.

Family 17: When $a=0$ and $b=-c$, then $\Phi(\xi)=$ $-2 \arctan [1 / c(\xi+C)]$,

where $A_{k}, B_{k}(k=1,2, \ldots, m), a, b$, and $c$ are constants to be determined later. But the positive integer $m$ can be determined by considering the homogeneous balance between the highest order derivatives and nonlinear terms appearing in (6). If $m$ is not an integer, then a transformation formula should be used to overcome this difficulty.

Step 3. Substituting (5) into (4) with the value of $m$ obtained in Step 2, collecting the coefficients of $\tan (\Phi(\xi) / 2)^{k}, \cot (\Phi(\xi) / 2)^{k}(k=0,1,2, \ldots)$, and then setting each coefficient to zero, we can get a set of overdetermined partial differential equations for $A_{0}, A_{k}, B_{k}(k=1,2, \ldots, m) a, b, c$, and $p$ with the aid of symbolic computation Maple.
Step 4. Solve the algebraic equations in Step 3, and then substitute $A_{0}, A_{1}, B_{1}, \ldots, A_{m}, B_{m}, \mu, p$ in (5).

\section{The Generalized Fitzhugh-Nagumo Equation}

We consider the generalized Fitzhugh-Nagumo equation with time-dependent coefficients as follows:

$$
\begin{aligned}
u_{t}+\alpha(t) u_{x}-\beta(t) u_{x x} \\
\quad+\gamma(t)\left(\rho u-(1+\rho) u^{2}+u^{3}\right)=0,
\end{aligned}
$$

where $\alpha(t), \beta(t)$, and $\gamma(t)$ are arbitrary functions of $t$. By using the wave variable $\xi=\mu[x-c(t) t]$, reduce it to an ODE as follows:

$$
\begin{gathered}
\mu\left(\alpha(t)-t \frac{d c(t)}{d t}-c(t)\right) u^{\prime}-\mu^{2} \beta(t) u^{\prime \prime} \\
+\gamma(t)\left(\rho u-(1+\rho) u^{2}+u^{3}\right)=0
\end{gathered}
$$

where $u^{\prime}=d u / d \xi$ and $u^{\prime \prime}=d^{2} u / d \xi^{2}$. Balancing the the linear term of the highest order $u^{\prime \prime}$ with the highest order nonlinear term $u^{3}$ by using homogenous principal, we have

$$
M+2=3 M, \quad M=1 .
$$

Then, the trail solutions are

$$
\begin{aligned}
u(\xi)= & A_{0}+A_{1}\left[p+\tan \left(\frac{\Phi(\xi)}{2}\right)\right] \\
& +B_{1}\left[p+\tan \left(\frac{\Phi(\xi)}{2}\right)\right]^{-1}
\end{aligned}
$$

Substituting (10) and (6) into (8) and collecting all terms with the same order of $\tan (\Phi(\xi) / 2)$ together, the left-hand side of $(10)$ is converted into polynomial in $\tan (\Phi(\xi) / 2)$. Setting each coefficient of each polynomial to zero, we derive a set of algebraic equations for $a, b, c, \mu, c(t), p, A_{0}, A_{1}$, and $B_{1}$ as follows:

$$
\begin{aligned}
& \left(\tan \left(\frac{\Phi(\xi)}{2}\right)\right)^{0}: 2 \gamma(t) A_{1}^{3} p^{6}-2 \gamma(t) A_{1}^{2} p^{5}+6 \gamma(t) \\
& \cdot A_{0} A_{1}^{2} p^{5}-2 \gamma(t) \rho A_{1}^{2} p^{5}-4 \gamma(t) \rho A_{0} A_{1} p^{4} \\
& +6 \gamma(t) A_{1}^{2} B_{1} p^{4}+6 \gamma(t) A_{0}^{2} A_{1} p^{4}+2 \gamma(t) \rho A_{1} p^{4} \\
& \quad-4 \gamma(t) A_{0} A_{1} p^{4}-\mu t \frac{d c(t)}{d t} A_{1} b p^{3}-4 \gamma(t) A_{1} B_{1} p^{3}
\end{aligned}
$$




$$
\begin{aligned}
& +2 \gamma(t) * A_{0}^{3} p^{3}-\mu t \frac{d c(t)}{d t} A_{1} c p^{3}-2 \gamma(t) A_{0}^{2} p^{3} \\
& +2 \gamma(t) \rho A_{0} p^{3}-4 \gamma(t) \rho A_{1} B_{1} p^{3}-\mu c(t) A_{1} b p^{3} \\
& +12 \gamma(t) A_{0} A_{1} B_{1} p^{3}-\mu c(t) A_{1} c p^{3}-\mu^{2} \beta(t) \\
& \text { - } A_{1} a b p^{3}-2 \gamma(t) \rho A_{0}^{2} p^{3}+\mu \alpha(t) A_{1} b p^{3}-\mu^{2} \beta(t) \\
& \text { - } A_{1} a c p^{3}+\mu \alpha(t) A_{1} c p^{3}-4 \gamma(t) A_{0} B_{1} p^{2}+6 \gamma(t) \\
& \text { - } A_{0}^{2} B_{1} p^{2}-4 \gamma(t) \rho A_{0} B_{1} p^{2}+6 \gamma(t) A_{1} B_{1}^{2} p^{2} \\
& +2 \gamma(t) \rho B_{1} p^{2}-2 \gamma(t) B_{1}^{2} p-\mu \alpha(t) B_{1} c p+\mu^{2} \beta(t) \\
& \cdot B_{1} a b p+\mu c(t) B_{1} c p+\mu t \frac{d c(t)}{d t} B_{1} b p-\mu \alpha(t) B_{1} b p \\
& +\mu c(t) B_{1} b p-2 \gamma(t) \rho B_{1}^{2} p+6 \gamma(t) A_{0} B_{1}^{2} p+\mu t \\
& \cdot \frac{d c(t)}{d t} B_{1} c p+\mu^{2} \beta(t) B_{1} a c p-\mu^{2} \beta(t) B_{1} b^{2} \\
& -\mu^{2} \beta(t) B_{1} c^{2}-2 \mu^{2} \beta(t) B_{1} b c+2 \gamma(t) B_{1}^{3}, \\
& \left(\tan \left(\frac{\Phi(\xi)}{2}\right)\right)^{1}: 12 \gamma(t) A_{1}^{3} p^{5}-10 \gamma(t) \rho A_{1}^{2} p^{4} \\
& +30 \gamma(t) A_{0} A_{1}^{2} p^{4}-10 \gamma(t) A_{1}^{2} p^{4}-2 \mu c(t) A_{1} a p^{3} \\
& -\mu^{2} \beta(t) A_{1} c^{2} p^{3}+8 \gamma(t) \rho A_{1} p^{3}+2 \mu \alpha(t) A_{1} a p^{3} \\
& +\mu^{2} \beta(t) A_{1} b^{2} p^{3}-16 \gamma(t) \rho A_{0} A_{1} p^{3}-16 \gamma(t) \\
& \text { - } A_{0} A_{1} p^{3}-2 \mu^{2} \beta(t) A_{1} a^{2} p^{3}-2 \mu t \frac{d c(t)}{d t} A_{1} a p^{3} \\
& +24 \gamma(t) A_{1}^{2} B_{1} p^{3}+24 \gamma(t) A_{0}^{2} A_{1} p^{3}-3 \mu c(t) \\
& \text { - } A_{1} b p^{2}-3 \mu^{2} \beta(t) A_{1} a b p^{2}-3 \mu t \frac{d c(t)}{d t} A_{1} c p^{2} \\
& +6 \gamma(t) A_{0}^{3} p^{2}-3 \mu c(t) A_{1} c p^{2}+3 \mu \alpha(t) A_{1} b p^{2} \\
& +3 \mu \alpha(t) A_{1} c p^{2}-12 \gamma(t) A_{1} B_{1} p^{2}+6 \gamma(t) \rho A_{0} p^{2} \\
& -3 \mu t \frac{d c(t)}{d t} A_{1} b p^{2}-12 \gamma(t) \rho A_{1} B_{1} p^{2}-3 \mu^{2} \beta(t) \\
& \text { - } A_{1} a c p^{2}+36 \gamma(t) A_{0} A_{1} B_{1} p^{2}-6 \gamma(t) \rho A_{0}^{2} p^{2} \\
& -6 \gamma(t) A_{0}^{2} p^{2}+12 \gamma(t) A_{0}^{2} B_{1} p+2 \mu t \frac{d c(t)}{d t} B_{1} a p \\
& -8 \gamma(t) \rho A_{0} B_{1} p+2 \mu c(t) B_{1} a p+\mu^{2} \beta(t) B_{1} c^{2} p \\
& +2 \mu^{2} \beta(t) B_{1} a^{2} p+4 \gamma(t) \rho B_{1} p-\mu^{2} \beta(t) B_{1} b^{2} p \\
& -2 \mu \alpha(t) B_{1} a p+12 \gamma(t) A_{1} B_{1}^{2} p-8 \gamma(t) A_{0} B_{1} p \\
& +\mu t 4 \gamma(t) \rho B_{1} p-\mu^{2} \beta(t) B_{1} b^{2} p-B_{1} b-\mu \alpha(t) B_{1} c \\
& +6 \gamma(t) A_{0} B_{1}^{2}-2 \gamma(t) B_{1}^{2}-2 \gamma(t) \rho B_{1}^{2}+\mu c(t) B_{1} b \\
& -\mu \alpha(t) B_{1} b+\mu t 4 \gamma(t) \rho B_{1} p-\mu^{2} \beta(t) B_{1} b^{2} p-B_{1} c \\
& -3 \mu^{2} \beta(t) B_{1} a c-3 \mu^{2} \beta(t) B_{1} a b+\mu c(t) B_{1} c, \\
& \left(\tan \left(\frac{\Phi(\xi)}{2}\right)\right)^{2}: 30 \gamma(t) A_{1}^{3} p^{4}-\mu \alpha(t) A_{1} b p^{3}-\mu t \\
& \cdot \frac{d c(t)}{d t} A_{1} c p^{3}-3 \mu^{2} \beta(t) A_{1} a c p^{3}+\mu t \frac{d c(t)}{d t} A_{1} b p^{3} \\
& -20 \gamma(t) \rho A_{1}^{2} p^{3}-\mu c(t) A_{1} c p^{3}-20 \gamma(t) A_{1}^{2} p^{3} \\
& +\mu c(t) A_{1} b p^{3}+\mu \alpha(t) A_{1} c p^{3}+60 \gamma(\mathrm{t}) A_{0} A_{1}^{2} p^{3} \\
& +3 \mu^{2} \beta(t) A_{1} a b p^{3}-24 \gamma(t) \rho A_{0} A_{1} p^{2}-6 \mu t 20 \gamma(t) \\
& \cdot A_{1}^{2} p^{3}+\mu c(t) A_{1} b p^{3}+\mu \alpha(t) A_{1} c p^{3}+60 \gamma(t) \\
& \text { - } A_{0} A_{1}^{2} p^{3}+A_{1} a p^{2}-6 \mu c(t) A_{1} a p^{2}-3 \mu^{2} \beta(t) \\
& \text { - } A_{1} c^{2} p^{2}+36 \gamma(t) A_{1}^{2} B_{1} p^{2}-6 \mu^{2} \beta(t) A_{1} a^{2} p^{2} \\
& +3 \mu^{2} \beta(t) A_{1} b^{2} p^{2}-24 \gamma(t) A_{0} A_{1} p^{2}+12 \gamma(t) \\
& \text { - } \rho A_{1} p^{2}+36 \gamma(t) A_{0}^{2} A_{1} p^{2}+6 \mu \alpha(t) A_{1} a p^{2}+\mu t \\
& \cdot \frac{d c(t)}{d t} B_{1} c p-3 \mu^{2} \beta(t) A_{1} a b p+36 \gamma(t) A_{0} A_{1} B_{1} p \\
& +\mu \alpha(t) B_{1} b p-\mu t \frac{d c(t)}{d t} B_{1} b p-\mu c(t) B_{1} b p-6 \gamma(t) \\
& \text { - } A_{0}^{2} p+3 \mu^{2} \beta(t) B_{1} a c p-3 \mu^{2} \beta(t) B_{1} a b p \\
& -3 \mu^{2} \beta(t) B_{1} a c p-12 \gamma(t) A_{1} B_{1} p-\mu \alpha(t) B_{1} c p \\
& +6 \gamma(t) A_{0}^{3} p+\mu c(t) B_{1} c p+3 \mu \alpha(t) A_{1} c p+3 \mu \alpha(t) \\
& \text { - } A_{1} b p+6 \gamma(t) \rho A_{0} p-3 \mu t \frac{d c(t)}{d t} A_{1} b p-3 \mu c(t) \\
& \text { - } A_{1} b p-3 \mu c(t) A_{1} c p-12 \gamma(t) \rho A_{1} B_{1} p-6 \gamma(t) \\
& \cdot \rho A_{0}^{2} p-3 \mu t \frac{d c(t)}{d t} A_{1} c p-2 \mu \alpha(t) B_{1} a-4 \lambda(t) \\
& \text { - } A_{0} B_{1}+2 \gamma(t) \rho B_{1}+6 \gamma(t) A_{0}^{2} B_{1}+\mu^{2} \beta(t) B_{1} b^{2} \\
& +6 \gamma(t) A_{1} B_{1}^{2}-2 \mu^{2} \beta(t) B_{1} a^{2}+2 \mu t \frac{d c(t)}{d t} B_{1} a \\
& -4 \gamma(t) \rho A_{0} B_{1}+2 \mu c(t) B_{1} a-\mu^{2} \beta(t) B_{1} c^{2}, \\
& \left(\tan \left(\frac{\Phi(\xi)}{2}\right)\right)^{3}: 2 \mu^{2} \beta(t) A_{1} b c p^{3}-\mu^{2} \beta(t) A_{1} c^{2} p^{3} \\
& -\mu^{2} \beta(t) A_{1} b^{2} p^{3}+40 \gamma(t) A_{1}^{3} p^{3}-3 \mu c(t) A_{1} c p^{2} \\
& +60 \gamma(t) A_{0} A_{1}^{2} p^{2}+3 \mu c(t) A_{1} b p^{2}-3 \mu \alpha(t) A_{1} b p^{2} \\
& +3 \mu t \frac{d c(t)}{d t} A_{1} b p^{2}-9 \mu^{2} \beta(t) A_{1} a c p^{2}+3 \mu \alpha(t)
\end{aligned}
$$




$$
\begin{aligned}
& \cdot A_{1} c p^{2}-3 \mu t \frac{d c(t)}{d t} A_{1} c p^{2}+9 \mu^{2} \beta(t) A_{1} a b p^{2} \\
& -20 \gamma(t) A_{1}^{2} p^{2}-20 \gamma(t) \rho A_{1}^{2} p^{2}-3 \mu^{2} \beta(t) A_{1} c^{2} p \\
& -6 \mu^{2} \beta(t) A_{1} a^{2} p+8 A_{1} \gamma(t) \rho p+6 \mu \alpha(t) A_{1} a p \\
& -2 \mu^{2} \beta(t) B_{1} b c p-16 \gamma(t) \rho A_{0} A_{1} p+3 \mu^{2} \beta(t) \\
& \cdot A_{1} b^{2} p-6 \mu c(t) A_{1} a p-6 \mu t \frac{d c(t)}{d t} A_{1} a p \\
& -16 A_{1} \gamma(t) A_{0} p+\mu^{2} \beta(t) B_{1} c^{2} p+\mu^{2} \beta(t) B_{1} b^{2} p \\
& +24 \gamma(t) A_{0}^{2} A_{1} p+24 \gamma(t) A_{1}^{2} B_{1} p-4 \gamma(t) \rho A_{1} B_{1} \\
& -\mu t \frac{d c(t)}{d t} A_{1} c-\mu^{2} \beta(t) B_{1} a c-\mu c(t) B_{1} b-\mu c(t) \\
& \text { - } A_{1} b+\mu t \frac{d c(t)}{d t} B_{1} c+2 \gamma(t) A_{0}^{3}+\mu \alpha(t) A_{1} c \\
& +12 \gamma(t) A_{0} A_{1} B_{1}-\mu c(t) A_{1} c+\mu \alpha(t) A_{1} b \\
& +2 \gamma(t) \rho A_{0}-2 \gamma(t) A_{0}^{2}-\mu \alpha(t) B_{1} c-\mu^{2} \beta(t) A_{1} a c \\
& -4 \gamma(t) A_{1} B_{1}-\mu t \frac{d c(t)}{d t} B_{1} b+\mu^{2} \beta(t) B_{1} a b \\
& -\mu^{2} \beta(t) A_{1} a b-\mu t \frac{d c(t)}{d t} A_{1} b+\mu \alpha(t) B_{1} b+\mu c(t) \\
& \cdot B_{1} c-2 \gamma(t) \rho A_{0}^{2} \\
& \left(\tan \left(\frac{\Phi(\xi)}{2}\right)\right)^{4}: A_{1}\left(3 \mu^{2} \beta(t) b^{2} p^{2}+3 \mu^{2} \beta(t) c^{2} p^{2}\right. \\
& -6 \mu^{2} \beta(t) b c p^{2}-30 \gamma(t) A_{1}^{2} p^{2}+3 \mu t \frac{d c(t)}{d t} c p \\
& +3 \mu c(t) c p-3 \mu \alpha(t) c p-3 \mu t \frac{d c(t)}{d t} b p \\
& +10 A_{1} \gamma(t) p-30 A_{1} \gamma(t) A_{0} p+3 \mu \alpha(t) b p \\
& +9 \mu^{2} \beta(t) a c p-9 \mu^{2} \beta(t) a b p+10 A_{1} \gamma(t) \rho p \\
& -3 \mu c(t) b p-6 \gamma(t) A_{0}^{2}-2 \gamma(t) \rho+4 \gamma(t) \rho A_{0} \\
& +2 \mu t \frac{d c(t)}{d t} a-2 \mu \alpha(t) a-6 \gamma(t) A_{1} B_{1}+2 \mu c(t) a \\
& +\mu \alpha(t) c-\mu c(t) c) \\
& \left(\tan \left(\frac{\Phi(\xi)}{2}\right)\right)^{6}:-\mu^{2} \beta(t) A_{1} c^{2}+2 \mu^{2} \beta(t) A_{1} b c \\
& +2 \gamma(t) A_{1}^{3}-\mu^{2} \beta(t) A_{1} b^{2} . \\
& a=a, \\
& b=c \\
& c=c \text {, } \\
& A_{0}=0 \\
& A_{1}=0 \text {, } \\
& B_{1}=p-\frac{c}{a}, \\
& \mu=\frac{1}{a} \sqrt{\frac{\gamma(t)}{2 \beta(t)}} \\
& p=p \\
& c(t)=\frac{1}{t} \int(\alpha(t) \\
& \left.+(1-2 \rho) \sqrt{\frac{\gamma(t) \beta(t)}{2}}\right) d t \\
& +C_{1} \text {, } \\
& u(\xi)=B_{1}\left[p+\tan \left(\frac{\Phi(\xi)}{2}\right)\right]^{-1}
\end{aligned}
$$$$
\left.+2 \mu^{2} \beta(t) a^{2}-\mu^{2} \beta(t) b^{2}+\mu^{2} \beta(t) c^{2}+4 \gamma(t) A_{0}\right),
$$$$
\left(\tan \left(\frac{\Phi(\xi)}{2}\right)\right)^{5}: A_{1}\left(12 \gamma(t) A_{1}^{2} p-3 \mu^{2} \beta(t) c^{2} p\right.
$$$$
-3 \mu^{2} \beta(t) b^{2} p+6 \mu^{2} \beta(t) b c p-2 \gamma(t) A_{1}
$$$$
+6 \gamma(t) A_{0} A_{1}-2 \gamma(t) \rho A_{1}+\mu c(t) b+3 \mu^{2} \beta(t) a b
$$$$
+\mu t \frac{d c(t)}{d t} b-\mu \alpha(t) b-3 \mu^{2} \beta(t) a c-\mu t \frac{d c(t)}{d t} c
$$

where $a, b$, and $c$ are arbitrary constants. By using (13), Families 7, 9, and 13 can be written, respectively, as

$$
\begin{gathered}
u_{1}(\xi)=\left[1+\frac{1}{p} \tan \left(\frac{1}{2}\right.\right. \\
\left.\left.\cdot \arctan \left[\frac{2 e^{a(\xi+C)}}{e^{2 a(\xi+C)}+1}, \frac{e^{2 a(\xi+C)}-1}{e^{2 a(\xi+C)}+1}\right]\right)\right]^{-1},
\end{gathered}
$$




$$
\begin{aligned}
& u_{2}(\xi)=\left[1+\frac{1}{p-1} e^{k a(\xi+C)}\right]^{-1}, \\
& u_{3}(\xi)=\left[1-\frac{2 a}{p-1} e^{a(\xi+C)}\right]^{-1},
\end{aligned}
$$

where $\xi=(1 / a) \sqrt{\gamma(t) / 2 \beta(t)}\left(x-\int(\alpha(t)+(1-\right.$ $\left.2 \rho) \sqrt{\gamma(t) \beta(t) / 2}) d t+C_{1}\right)$.

Set 2. We have the following:

$$
\begin{aligned}
& a=a, \\
& b=c, \\
& c=c, \\
& A_{0}=0 \text {, } \\
& A_{1}=0 \text {, } \\
& B_{1}=\left(p-\frac{c}{a}\right) \rho, \\
& \mu=\frac{\rho}{a} \sqrt{\frac{\gamma(t)}{2 \beta(t)}}, \\
& p=p, \\
& c(t)=\frac{1}{t} \\
& \int\left(\alpha(t)+(\rho-2) \sqrt{\frac{\gamma(t) \beta(t)}{2}}\right) d t \\
& +C_{1} \text {, } \\
& u(\xi)=B_{1}\left[p+\tan \left(\frac{\Phi(\xi)}{2}\right)\right]^{-1} \text {, }
\end{aligned}
$$

where $a, b$, and $c$ are arbitrary constants. By using (17), Families 7, 9, and 13 can be written, respectively, as

$$
\begin{gathered}
u_{4}(\xi)=\rho\left[1+\frac{1}{p} \tan \left(\frac{1}{2}\right.\right. \\
\cdot \arctan \left[\frac{2 e^{a(\xi+C)}}{e^{2 a(\xi+C)}+1},\right. \\
\left.\left.\left.\frac{e^{2 a(\xi+C)}-1}{e^{2 a(\xi+C)}+1}\right]\right)\right]^{-1},
\end{gathered}
$$

$$
\begin{aligned}
& u_{5}(\xi)=\rho\left[1+\frac{1}{p-1} e^{k a(\xi+C)}\right]^{-1}, \\
& u_{6}(\xi)=\rho\left[1-\frac{2 a}{p+1} e^{a(\xi+C)}\right]^{-1},
\end{aligned}
$$

where $\xi=(\rho / a) \sqrt{\gamma(t) / 2 \beta(t)}\left(x-\int(\alpha(t)+(\rho-\right.$ 2) $\left.\sqrt{\gamma(t) \beta(t) / 2}) d t+C_{1}\right)$.

Set 3. We have the following:

$$
\begin{aligned}
& a=a, \\
& b=c, \\
& c=c, \\
& A_{0}=1, \\
& A_{1}=0, \\
& B_{1}=\left(-p+\frac{c}{a}\right), \\
& \mu=\frac{1}{a} \sqrt{\frac{\gamma(t)}{2 \beta(t)}}, \\
& p=p, \\
& c(t)=\frac{1}{t} \int(\alpha(t) \\
& \left.\quad+(1-2 \rho) \sqrt{\frac{\gamma(t) \beta(t)}{2}}\right) d t \\
& \quad+C_{1}, \\
& u(\xi)=1+B_{1}\left[p+\tan \left(\frac{\Phi(\xi)}{2}\right)\right]^{-1},
\end{aligned}
$$

where $a, b$, and $c$ are arbitrary constants. By using (20), Families 7, 9, and 13 can be written, respectively, as

$$
\begin{gathered}
u_{7}(\xi)=1-p\left[p+\tan \left(\frac{1}{2}\right.\right. \\
\cdot \arctan \left[\frac{2 e^{a(\xi+C)}}{e^{2 a(\xi+C)}+1},\right. \\
\left.\left.\left.\frac{e^{2 a(\xi+C)}-1}{e^{2 a(\xi+C)}+1}\right]\right)\right]^{-1}
\end{gathered}
$$




$$
\begin{aligned}
& u_{8}(\xi)=1+\left[1+\frac{1}{p-1} e^{k a(\xi+C)}\right]^{-1}, \\
& u_{9}(\xi)=1-\left[1-\frac{2 a}{p+1} e^{a(\xi+C)}\right]^{-1},
\end{aligned}
$$

where $\xi=(1 / a) \sqrt{\gamma(t) / 2 \beta(t)}\left(x-\int(\alpha(t)+(1-\right.$ $\left.2 \rho) \sqrt{\gamma(t) \beta(t) / 2}) d t+C_{1}\right)$.

Set 4. We have the following:

$$
\begin{aligned}
& a=a, \\
& b=c \text {, } \\
& c=c \text {, } \\
& A_{0}=1 \text {, } \\
& A_{1}=0 \text {, } \\
& B_{1}=(\rho-1)\left(p-\frac{c}{a}\right), \\
& \mu=\frac{\rho-1}{a} \sqrt{\frac{\gamma(t)}{2 \beta(t)}}, \\
& p=p \\
& c(t)=\frac{1}{t} \\
& \int(\alpha(t)+(\rho+1) \sqrt{2 \gamma(t) \beta(t)}) d t \\
& +C_{1} \text {, } \\
& u(\xi)=1+B_{1}\left[p+\tan \left(\frac{\Phi(\xi)}{2}\right)\right]^{-1}
\end{aligned}
$$

where $a, b$, and $c$ are arbitrary constants. By using (23), Families 7, 9, and 13 can be written, respectively, as

$$
\begin{gathered}
u_{10}(\xi)=1-(\rho-1)\left[1+\frac{1}{p} \tan \left(\frac{1}{2}\right.\right. \\
\cdot \arctan \left[\frac{2 e^{a(\xi+C)}}{e^{2 a(\xi+C)}+1},\right. \\
\left.\left.\left.\frac{e^{2 a(\xi+C)}-1}{e^{2 a(\xi+C)}+1}\right]\right)\right]^{-1},
\end{gathered}
$$

$$
\begin{gathered}
u_{11}(\xi)=1+(\rho-1)\left[1+\frac{1}{p-1}\right. \\
\left.\cdot e^{k a(\xi+C)}\right]^{-1}
\end{gathered}
$$$$
u_{12}(\xi)=1+(\rho-1)\left[1-\frac{2 a}{p+1}\right.
$$

$$
\left.\cdot e^{a(\xi+C)}\right]^{-1}
$$

where $\xi=((\rho-1) / a) \sqrt{\gamma(t) / 2 \beta(t)}\left(x-\int(\alpha(t)+(\rho+\right.$ 1) $\left.\sqrt{2 \gamma(t) \beta(t)}) d t+C_{1}\right)$.

Set 5. We have the following:

$$
\begin{aligned}
& a=a, \\
& b=c, \\
& c=c, \\
& A_{0}=\rho, \\
& A_{1}=0, \\
& B_{1}=\rho\left(-p+\frac{c}{a}\right), \\
& \mu=\frac{\rho}{a} \sqrt{\frac{\gamma(t)}{2 \beta(t)}}, \\
& p=p, \\
& c(t)=\frac{1}{t} \\
& \quad . \int(\alpha(t)+(2-\rho) \sqrt{2 \gamma(t) \beta(t)}) d t \\
& \quad+C_{1}, \\
& u(\xi)=\rho+B_{1}\left[p+\tan \left(\frac{\Phi(\xi)}{2}\right)\right]^{-1},
\end{aligned}
$$

where $a, b$, and $c$ are arbitrary constants. By using (26), Families 7, 9, and 13 can be written, respectively, as

$$
\begin{gathered}
u_{13}(\xi)=\rho-p \rho\left[p+\tan \left(\frac{1}{2}\right.\right. \\
\cdot \arctan \left[\frac{2 e^{a(\xi+C)}}{e^{2 a(\xi+C)}+1},\right. \\
\left.\left.\left.\frac{e^{2 a(\xi+C)}-1}{e^{2 a(\xi+C)}+1}\right]\right)\right]^{-1}
\end{gathered}
$$




$$
\begin{array}{ll}
u_{14}(\xi)=\rho-\rho\left[1+\frac{1}{p-1} e^{k a(\xi+C)}\right]^{-1}, & u_{18}(\xi)=\rho-(\rho-1)\left[1-\frac{2 a}{p+1}\right. \\
u_{15}(\xi)=\rho-\rho\left[1-\frac{2 a}{p+1} e^{a(\xi+C)}\right]^{-1}, & \left.\cdot e^{a(\xi+C)}\right]^{-1},
\end{array}
$$

where $\xi=(\rho / a) \sqrt{\gamma(t) / 2 \beta(t)}\left(x-\int(\alpha(t)+(2-\right.$ ค) $\left.\sqrt{2 \gamma(t) \beta(t)}) d t+C_{1}\right)$.

Set 6. We have the following:

$$
\begin{aligned}
& a=a, \\
& b=c \\
& c=c \\
& A_{0}=\rho, \\
& A_{1}=0 \\
& B_{1}=(\rho-1)\left(-p+\frac{c}{a}\right), \\
& \mu=\frac{\rho-1}{a} \sqrt{\frac{\gamma(t)}{2 \beta(t)}}, \\
& p=p, \\
& c(t)=\frac{1}{t} \\
& \quad \cdot \int(\alpha(t)-(\rho+1) \sqrt{2 \gamma(t) \beta(t)}) d t \\
& \quad+C_{1}, \\
& u(\xi)=\rho+B_{1}\left[p+\tan \left(\frac{\Phi(\xi)}{2}\right)\right]^{-1},
\end{aligned}
$$

where $a, b$, and $c$ are arbitrary constants. By using (29), Families 7, 9, and 13 can be written, respectively, as

$$
\begin{gathered}
u_{16}(\xi)=\rho-(\rho-1)\left[1+\frac{1}{p} \tan \left(\frac{1}{2}\right.\right. \\
\cdot \arctan \left[\frac{2 e^{a(\xi+C)}}{e^{2 a(\xi+C)}+1},\right. \\
\left.\left.\left.\frac{e^{2 a(\xi+C)}-1}{e^{2 a(\xi+C)}+1}\right]\right)\right]^{-1}, \\
u_{17}(\xi)=\rho-(\rho-1)\left[1+\frac{1}{p-1}\right. \\
\left.\cdot e^{k a(\xi+C)}\right]^{-1}
\end{gathered}
$$

where $\xi=((\rho-1) / a) \sqrt{\gamma(t) / 2 \beta(t)}\left(x-\int(\alpha(t)-(\rho+\right.$ 1) $\left.\sqrt{2 \gamma(t) \beta(t)}) d t+C_{1}\right)$.

Set 7. We have the following:

$$
\begin{aligned}
& A_{0}=\frac{1 \pm i p}{2}, \\
& A_{1}=0 \text {, } \\
& B_{1}= \pm \frac{1+p^{2}}{2} i \\
& \mu= \pm \frac{1}{c B_{1}} \sqrt{\frac{\gamma(t)}{2 \beta(t)}}\left[\left(p A_{0}+B_{1}\right)^{2}+A_{0}\left(1+p^{2}\right)\right. \\
& \left.+A_{0}^{2}-p B_{1}\right], \\
& b \neq c \text {, } \\
& c=c \text {, } \\
& a^{2}+b^{2}-c^{2} \\
& =\frac{c^{2} B_{1}^{2}}{\left(\left(p A_{0}+B_{1}\right)^{2}+A_{0}\left(1+p^{2}\right)+A_{0}^{2}-p B_{1}\right)^{2}}, \\
& p=p \\
& c(t)=\frac{1}{t} \int\left(\alpha(t)+(2 \rho-1) \sqrt{\frac{\gamma(t) \beta(t)}{2}}\right) d t \\
& +C_{1} \text {, } \\
& u(\xi)=A_{0}+B_{1}\left[p+\tan \left(\frac{\Phi(\xi)}{2}\right)\right]^{-1},
\end{aligned}
$$

where $a, b$, and $c$ are arbitrary constants. By using (32), Family 2 can be written as

$$
\begin{aligned}
& u_{19}(\xi)=\frac{1 \pm i p}{2} \pm \frac{1+p^{2}}{2} i\left[p+\frac{a}{b-c}\right. \\
& +\frac{\sqrt{b^{2}+a^{2}-c^{2}}}{b-c} \\
& \left.\cdot \tanh \left(\frac{\sqrt{b^{2}+a^{2}-c^{2}}}{2}(\xi+C)\right)\right]^{-1}
\end{aligned}
$$


where $\xi= \pm\left(1 / c B_{1}\right) \sqrt{\gamma(t) / 2 \beta(t)}\left[\left(p A_{0}+B_{1}\right)^{2}+A_{0}\left(1+p^{2}\right)+\quad\right.$ Set 8 . We have the following: $\left.A_{0}^{2}-p B_{1}\right]\left(x-\int(\alpha(t)+(2 \rho-1) \sqrt{\gamma(t) \beta(t) / 2}) d t+C_{1}\right)$.

$$
\begin{aligned}
A_{0} & =\frac{1 \pm i p}{2} \rho, \\
A_{1} & =0 \\
B_{1} & = \pm \frac{1+p^{2}}{2} \rho i, \\
\mu & = \pm \frac{1}{c B_{1}} \sqrt{\frac{\gamma(t)}{2 \beta(t)}}\left[\left(p A_{0}+B_{1}\right)^{2}+\rho A_{0}\left(1+p^{2}\right)+A_{0}^{2}-\rho p B_{1}\right], \\
b & \neq c \\
c & =c \\
a^{2}+b^{2}-c^{2} & =\frac{\left[\left(p A_{0}+B_{1}\right)^{2}+\rho A_{0}\left(1+p^{2}\right)+A_{0}^{2}-\rho p B_{1}\right]^{2}}{c^{2} B_{1}^{2}} \\
p & =p \\
c(t) & =\frac{1}{t} \int\left(\alpha(t)+(\rho-2) \sqrt{\frac{\gamma(t) \beta(t)}{2}}\right) d t+C_{1}, \\
u(\xi) & =A_{0}+B_{1}\left[p+\tan \left(\frac{\Phi(\xi)}{2}\right)\right]^{-1},
\end{aligned}
$$

where $a, b$, and $c$ are arbitrary constants. By using (35), Family 2 can be written as

$$
\begin{gathered}
u_{19}(\xi)=\frac{1 \pm i p}{2} \rho \pm \frac{1+p^{2}}{2} \rho i[p \\
+\frac{a}{b-c}+\frac{\sqrt{b^{2}+a^{2}-c^{2}}}{b-c}
\end{gathered}
$$

$$
\begin{aligned}
A_{0} & =\frac{(\rho+1) \pm i p(\rho-1)}{2}, \\
A_{1} & =0, \\
B_{1} & = \pm \frac{\left(1+p^{2}\right)(\rho-1)}{p} i, \\
a^{2}+b^{2}-c^{2} & =\frac{c^{2} B_{1}^{2}}{\left[\left(p A_{0}+B_{1}\right)^{2}+\rho A_{0}\left(1+p^{2}\right)+A_{0}^{2}-\rho p B_{1}\right]^{2}},
\end{aligned}
$$

where $\xi= \pm\left(1 / c B_{1}\right) \sqrt{\gamma(t) / 2 \beta(t)}\left[\left(p A_{0}+B_{1}\right)^{2}+\rho A_{0}\left(1+p^{2}\right)+\right.$ $\left.A_{0}^{2}-\rho p B_{1}\right]\left(x-\int(\alpha(t)+(\rho-2) \sqrt{\gamma(t) \beta(t) / 2}) d t+C_{1}\right)$.

Set 9. We have the following: 


$$
\begin{aligned}
b & \neq c, \\
c & =c \\
\mu & = \pm \frac{1}{c B_{1}} \sqrt{\frac{\gamma(t)}{2 \beta(t)}}\left[(\rho+1)\left(A_{0} p^{2}+p B_{1}+A_{0}\right)-\left(p A_{0}+B_{1}\right)^{2}-A_{0}^{2}-\rho\right], \\
p & =p, \\
c(t) & =\frac{1}{t} \int\left(\alpha(t)+(\rho+1) \sqrt{\frac{\gamma(t) \beta(t)}{2}}\right) d t+C_{1}, \\
u(\xi) & =A_{0}+B_{1}\left[p+\tan \left(\frac{\Phi(\xi)}{2}\right)\right]^{-1},
\end{aligned}
$$

where $a, b$, and $c$ are arbitrary constants. By using (38), Family 2 can be written as

$$
\begin{aligned}
& u_{19}(\xi)=\frac{(\rho+1) \pm i p(\rho-1)}{2} \\
& \pm \frac{\left(1+p^{2}\right)(\rho-1)}{p} i\left[p+\frac{a}{b-c}\right. \\
& +\frac{\sqrt{b^{2}+a^{2}-c^{2}}}{b-c} \\
& \left.\cdot \tanh \left(\frac{\sqrt{b^{2}+a^{2}-c^{2}}}{2}(\xi+C)\right)\right]^{-1},
\end{aligned}
$$

where $\xi= \pm\left(1 / c B_{1}\right) \sqrt{\gamma(t) / 2 \beta(t)}\left[(\rho+1)\left(A_{0} p^{2}+p B_{1}+A_{0}\right)-\right.$ $\left.\left(p A_{0}+B_{1}\right)^{2}-A_{0}^{2}-\rho\right]\left(x-\int(\alpha(t)+(\rho+1) \sqrt{\gamma(t) \beta(t) / 2}) d t+C_{1}\right)$.

Set 10. We have the following:

$$
\begin{aligned}
A_{0} & =0, \\
A_{1} & =0, \\
B_{1} & = \pm \frac{2 \rho}{\rho-1} i, \\
a^{2} & +b^{2}-c^{2} \\
& =\frac{c^{2} B_{1}^{2}(\rho-1)^{2}}{\left[B_{1}^{2}-p B_{1}+\rho\left(1-p B_{1}+p^{2}\right)\right]^{2}},
\end{aligned}
$$

$$
\begin{aligned}
& p= \pm \frac{\rho+1}{\rho-1} i, \\
& b \neq c, \\
& c=c, \\
& \mu= \pm \frac{1}{c B_{1}} \sqrt{\frac{\gamma(t)}{2 \beta(t)}}\left[B_{1}^{2}-p B_{1}+\rho(1\right. \\
& \left.\left.-p B_{1}+p^{2}\right)\right] \text {, } \\
& c(t)=\frac{1}{t} \int(\alpha(t) \\
& \left.-(\rho+1) \sqrt{\frac{\gamma(t) \beta(t)}{2}}\right) d t+C_{1}, \\
& u(\xi)=B_{1}\left[p+\tan \left(\frac{\Phi(\xi)}{2}\right)\right]^{-1},
\end{aligned}
$$

where $a, b$, and $c$ are arbitrary constants. By using (41), Family 2 can be written as

$$
\begin{gathered}
u_{20}(\xi)= \pm \frac{2 \rho}{\rho-1} i\left[ \pm \frac{\rho+1}{\rho-1} i+\frac{a}{b-c}\right. \\
+\frac{\sqrt{b^{2}+a^{2}-c^{2}}}{b-c} \\
\left.\cdot \tanh \left(\frac{\sqrt{b^{2}+a^{2}-c^{2}}}{2}(\xi+C)\right)\right]^{-1},
\end{gathered}
$$

where $\xi= \pm\left(1 / c B_{1}\right) \sqrt{\gamma(t) / 2 \beta(t)}\left[B_{1}^{2}-p B_{1}+\rho\left(1-p B_{1}+p^{2}\right)\right](x-$ $\left.\int(\alpha(t)-(\rho+1) \sqrt{\gamma(t) \beta(t) / 2}) d t+C_{1}\right)$. 
Set 11. We have the following:

$$
\begin{aligned}
& A_{0}=1 \text {, } \\
& A_{1}=0 \text {, } \\
& B_{1}=B_{1} \text {, } \\
& a^{2}+b^{2}-c^{2}=-\frac{c^{2}}{\left[\rho\left(1+p B_{1}+p^{2}\right)-1-\left(p+B_{1}\right)^{2}\right]^{2}}\left[B _ { 1 } \left(-12 p-6 \rho p^{3}-6 \rho^{2} p+2 \rho^{2} p^{3}\right.\right. \\
& \left.+p^{2} \rho^{2}+18 p \rho+4 p^{3}\right)+B_{1}^{2}\left(-6 \rho p^{2}-6+6 \rho+6 p^{2}\right)+B_{1}^{3}(-2 \rho p+4 p)+B_{1}^{4}+1+\rho^{2} \\
& \left.+12 \rho p^{2}-6 p^{2}-2 \rho+p^{4}-2 \rho^{2} p^{2}+\rho^{2} p^{4}-2 \rho p^{4}\right] \\
& p=-\frac{\rho-2}{2(\rho-1)} B_{1} \\
& b \neq c, \\
& c=c, \\
& \mu= \pm \frac{1}{c B_{1}} \sqrt{\frac{\gamma(t)}{2 \beta(t)}}\left[\rho\left(1+p B_{1}+p^{2}\right)-1-\left(p+B_{1}\right)^{2}\right] \\
& c(t)=\frac{1}{t} \int\left(\alpha(t)+(\rho-1) \sqrt{\frac{\gamma(t) \beta(t)}{2}}\right) d t+C_{1}, \\
& u(\xi)=1+B_{1}\left[p+\tan \left(\frac{\Phi(\xi)}{2}\right)\right]^{-1}
\end{aligned}
$$

where $a, b$, and $c$ are arbitrary constants. By using (44), Family 2 can be written as

$$
\begin{gathered}
u_{20}(\xi)=1+B_{1}\left[-\frac{\rho-2}{2(\rho-1)} B_{1}+\frac{a}{b-c}\right. \\
-\frac{\sqrt{c^{2}-b^{2}-a^{2}}}{b-c}
\end{gathered}
$$

$$
\left.\cdot \tan \left(\frac{\sqrt{c^{2}-b^{2}-a^{2}}}{2}(\xi+C)\right)\right]^{-1}
$$

where $\xi= \pm\left(1 / c B_{1}\right) \sqrt{\gamma(t) / 2 \beta(t)}\left[\rho\left(1+p B_{1}+p^{2}\right)-1-(p+\right.$ $\left.\left.B_{1}\right)^{2}\right]\left(x-\int(\alpha(t)+(\rho-1) \sqrt{\gamma(t) \beta(t) / 2}) d t+C_{1}\right)$.

Set 12. We have the following:

$$
\begin{aligned}
& A_{0}=\rho, \\
& A_{1}=0 \\
& B_{1}= \pm 2 \rho(\rho-1) i, \\
& a^{2}+b^{2}-c^{2}=\frac{c^{2}}{\left[\left(p \rho+B_{1}\right)^{2}-p b_{1}+\rho^{2}-\rho-\rho p^{2}\right]^{2}}\left[B _ { 1 } \left(2 p^{3} \rho+18 p \rho^{2}-6 p^{3} \rho^{2}-6 p \rho\right.\right.
\end{aligned}
$$




$$
\begin{aligned}
& \left.-12 p \rho^{3}+4 p^{3} \rho^{3}\right)+B_{1}^{2}\left(-6 \rho p^{2}+6 \rho+6 \rho^{2} p^{2}-6 \rho^{2}+p^{2}\right)+B_{1}^{3}(4 p \rho-2 p)+B_{1}^{4}+\left(p^{4}\right. \\
& \left.\left.-6 p^{2}\right)\left(\rho^{4}-2 \rho^{3}+\rho^{2}\right)+\rho^{2}(\rho-1)^{2}\right], \\
& p=\mp(2 \rho-1) i \\
& b \neq c, \\
& c=c, \\
& \mu= \pm \frac{1}{c B_{1}} \sqrt{\frac{\gamma(t)}{2 \beta(t)}}\left[\left(p \rho+B_{1}\right)^{2}-p b_{1}+\rho^{2}-\rho-\rho p^{2}\right] \\
& c(t)=\frac{1}{t} \int\left(\alpha(t)+(2 \rho-1) \sqrt{\frac{\gamma(t) \beta(t)}{2}}\right) d t+C_{1}, \\
& u(\xi)=\rho+B_{1}\left[p+\tan \left(\frac{\Phi(\xi)}{2}\right)\right]^{-1},
\end{aligned}
$$

where $a, b$, and $c$ are arbitrary constants. By using (47), Family 2 can be written as

$$
\begin{gathered}
u_{20}(\xi)=\rho \pm 2 \rho(\rho-1) i[\mp(2 \rho-1) i \\
+\frac{a}{b-c}+\frac{\sqrt{b^{2}+a^{2}-c^{2}}}{b-c} \\
\left.\cdot \tanh \left(\frac{\sqrt{b^{2}+a^{2}-c^{2}}}{2}(\xi+C)\right)\right]^{-1}
\end{gathered}
$$

where $\xi= \pm\left(1 / c B_{1}\right) \sqrt{\gamma(t) / 2 \beta(t)}\left[\left(p \rho+B_{1}\right)^{2}-p b_{1}+\rho^{2}-\rho-\right.$ $\left.\rho p^{2}\right]\left(x-\int(\alpha(t)+(2 \rho-1) \sqrt{\gamma(t) \beta(t) / 2}) d t+C_{1}\right)$.

Set 13. We have the following:

$$
\begin{aligned}
& a=\frac{1}{\mu} \sqrt{\frac{\gamma(t)}{2 \beta(t)}} \\
& b=-\frac{A_{0}-1}{p \mu} \sqrt{\frac{\gamma(t)}{2 \beta(t)}}, \\
& c=\frac{A_{0}-1}{p \mu} \sqrt{\frac{\gamma(t)}{2 \beta(t)}},
\end{aligned}
$$

$$
\begin{aligned}
& A_{0}=A_{0}, \\
& A_{1}=0, \\
& B_{1}=-p A_{0}, \\
& p=p \\
& \mu=\mu, \\
& c(t)=\frac{1}{t} \int(\alpha(t)
\end{aligned}
$$

$$
\left.-(2 \rho-1) \sqrt{\frac{\gamma(t) \beta(t)}{2}}\right) d t
$$

$+C_{1}$,

$$
u(\xi)=A_{0}+B_{1}\left[p+\tan \left(\frac{\Phi(\xi)}{2}\right)\right]^{-1}
$$

By using (50), Families 1, 2, 7, and 14 can be written, respectively, as

$$
u_{21}(\xi)=A_{0}-p A_{0}\left[p-\frac{p}{2\left(A_{0}-1\right)}+\frac{p}{2\left(A_{0}-1\right)} i \tan \left(\frac{1}{2 \mu} \sqrt{-\frac{\gamma(t)}{2 \beta(t)}}(\xi+C)\right)\right]^{-1}
$$




$$
\begin{aligned}
& u_{22}(\xi)=A_{0}-p A_{0}\left[p+\frac{p}{2\left(A_{0}-1\right)}-\frac{p}{2\left(A_{0}-1\right)} \tanh \left(\frac{1}{2 \mu} \sqrt{\frac{\gamma(t)}{2 \beta(t)}}(\xi+C)\right)\right]^{-1}, \\
& u_{23}(\xi) \\
& =A_{0} \\
& -p A_{0}\left[p+\tan \left(\frac{1}{2} \arctan \left[\frac{2 e^{(1 / \mu) \sqrt{\gamma(t) / 2 \beta(t)}(\xi+C)}}{e^{(2 / \mu) \sqrt{\gamma(t) / 2 \beta(t)(\xi+C)}+1}}, \frac{e^{(2 / \mu) \sqrt{\gamma(t) / 2 \beta(t)(\xi+C)}}-1}{e^{(2 / \mu) \sqrt{\gamma(t) / 2 \beta(t)(\xi+C)}}+1}\right]\right)\right]^{-1}, \\
& u_{24}(\xi)=A_{0}-p A_{0}\left[p-\left[\frac{(1 / \mu) \sqrt{\gamma(t) / 2 \beta(t)} e^{(1 / \mu) \sqrt{\gamma(t) / 2 \beta(t)}(\xi+C)}}{\left(\left(A_{0}-1\right) / p \mu\right) \sqrt{\gamma(t) / 2 \beta(t)} e^{(1 / \mu) \sqrt{\gamma(t) / 2 \beta(t)}(\xi+C)}-1}\right]\right]^{-1},
\end{aligned}
$$

where $\xi=\mu\left(x-\int(\alpha(t)-(2 \rho-1) \sqrt{\gamma(t) \beta(t) / 2}) d t+C_{1}\right)$.

Set 14. We have the following:

$$
\begin{aligned}
& a=\frac{\rho}{\mu} \sqrt{\frac{\gamma(t)}{2 \beta(t)}}, \\
& b=-\frac{\rho-A_{0}}{p \mu} \sqrt{\frac{\gamma(t)}{2 \beta(t)}}, \\
& c=\frac{\rho-A_{0}}{p \mu} \sqrt{\frac{\gamma(t)}{2 \beta(t)}}, \\
& A_{0}=A_{0}, \\
& A_{1}=0, \\
& B_{1}=-p A_{0},
\end{aligned}
$$

$$
\begin{aligned}
& p=p, \\
& \mu=\mu,
\end{aligned}
$$$$
c(t)=\frac{1}{t}
$$$$
\cdot \int\left(\alpha(t)-(\rho-2) \sqrt{\frac{\gamma(t) \beta(t)}{2}}\right) d t
$$$$
+C_{1} \text {, }
$$$$
u(\xi)=A_{0}+B_{1}\left[p+\tan \left(\frac{\Phi(\xi)}{2}\right)\right]^{-1}
$$

By using (50), Families 1, 2, 7, and 14 can be written, respectively, as

$$
\begin{aligned}
& u_{25}(\xi)=A_{0}-p A_{0}\left[p-\frac{p \rho}{2\left(\rho-A_{0}\right)}+\frac{p \rho}{2\left(\rho-A_{0}\right)} i \tan \left(\frac{\rho}{2 \mu} \sqrt{-\frac{\gamma(t)}{2 \beta(t)}}(\xi+C)\right)\right]^{-1}, \\
& u_{26}(\xi)=A_{0}-p A_{0}\left[p+\frac{p \rho}{2\left(\rho-A_{0}\right)}-\frac{p \rho}{2\left(\rho-A_{0}\right)} \tanh \left(\frac{\rho}{2 \mu} \sqrt{\frac{\gamma(t)}{2 \beta(t)}}(\xi+C)\right)\right]^{-1} \text {, } \\
& u_{27}(\xi) \\
& =A_{0}
\end{aligned}
$$

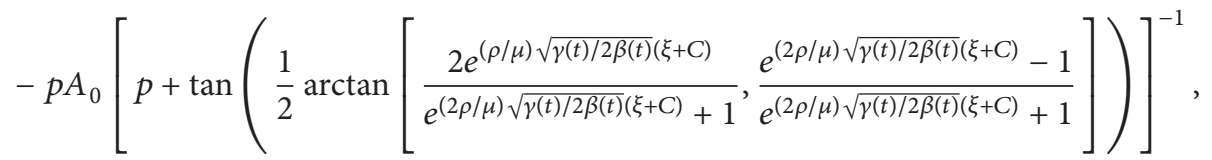

$$
\begin{aligned}
& u_{28}(\xi)=A_{0}-p A_{0}\left[p-\left[\frac{(\rho / \mu) \sqrt{\gamma(t) / 2 \beta(t)} e^{(\rho / \mu) \sqrt{\gamma(t) / 2 \beta(t)(\xi+C)}}}{\left(\left(\rho-A_{0}\right) / p \mu\right) \sqrt{\gamma(t) / 2 \beta(t)} e^{(\rho / \mu) \sqrt{\gamma(t) / 2 \beta(t)(\xi+C)}-1}}\right]\right]^{-1},
\end{aligned}
$$

where $\xi=\mu\left(x-\int(\alpha(t)-(\rho-2) \sqrt{\gamma(t) \beta(t) / 2}) d t+C_{1}\right)$. 
Set 15. We have the following:

$$
\begin{aligned}
& a=\frac{1}{\mu} \sqrt{\frac{\gamma(t)}{2 \beta(t)}}, \\
& b=-\frac{A_{0}}{p \mu} \sqrt{\frac{\gamma(t)}{2 \beta(t)}}, \\
& c=\frac{A_{0}}{p \mu} \sqrt{\frac{\gamma(t)}{2 \beta(t)}}, \\
& A_{0}=A_{0}, \\
& A_{1}=0, \\
& B_{1}=p-A_{0} p, \\
& p=p, \\
& \mu=\mu,
\end{aligned}
$$

$$
\begin{aligned}
& c(t)=\frac{1}{t} \int(\alpha(t) \\
& \left.-(2 \rho-1) \sqrt{\frac{\gamma(t) \beta(t)}{2}}\right) d t \\
& +C_{1}, \\
& u(\xi)=A_{0}+B_{1}\left[p+\tan \left(\frac{\Phi(\xi)}{2}\right)\right]^{-1} .
\end{aligned}
$$

By using (50), Families 1, 2, 7, and 14 can be written, respectively, as

$$
\begin{aligned}
& u_{29}(\xi)=A_{0}+\left(p-A_{0} p\right)\left[p-\frac{p}{2 A_{0}}+\frac{p}{2 A_{0}} i \tan \left(\frac{1}{2 \mu} \sqrt{-\frac{\gamma(t)}{2 \beta(t)}}(\xi+C)\right)\right]^{-1}, \\
& u_{30}(\xi)=A_{0}+\left(p-A_{0} p\right)\left[p+\frac{p}{2 A_{0}}-\frac{p}{2 A_{0}} \tanh \left(\frac{1}{2 \mu} \sqrt{\frac{\gamma(t)}{2 \beta(t)}}(\xi+C)\right)\right]^{-1}, \\
& u_{31}(\xi)=A_{0}+\left(p-A_{0} p\right) \\
& \cdot\left[p+\tan \left(\frac{1}{2} \arctan \left[\frac{2 e^{(1 / \mu) \sqrt{\gamma(t) / 2 \beta(t)}(\xi+C)}}{e^{(2 / \mu) \sqrt{\gamma(t) / 2 \beta(t)}(\xi+C)}+1}, \frac{e^{(2 / \mu) \sqrt{\gamma(t) / 2 \beta(t)}(\xi+C)}-1}{e^{(2 / \mu) \sqrt{\gamma(t) / 2 \beta(t)}(\xi+C)}+1}\right]\right)\right]^{-1}, \\
& u_{32}(\xi)=A_{0}+\left(p-A_{0} p\right)\left[p-\left[\frac{(1 / \mu) \sqrt{\gamma(t) / 2 \beta(t)} e^{(1 / \mu) \sqrt{\gamma(t) / 2 \beta(t)}(\xi+C)}}{\left(A_{0} / p \mu\right) \sqrt{\gamma(t) / 2 \beta(t)} e^{(1 / \mu) \sqrt{\gamma(t) / 2 \beta(t)}(\xi+C)}-1}\right]\right]^{-1},
\end{aligned}
$$

where $\xi=\mu\left(x-\int(\alpha(t)-(2 \rho-1) \sqrt{\gamma(t) \beta(t) / 2}) d t+C_{1}\right)$.

Set 16. We have the following:

$$
\begin{aligned}
& a=\frac{\rho-1}{\mu} \sqrt{\frac{\gamma(t)}{2 \beta(t)}}, \\
& b=-\frac{\rho-A_{0}}{p \mu} \sqrt{\frac{\gamma(t)}{2 \beta(t)}}, \\
& c=\frac{\rho-A_{0}}{p \mu} \sqrt{\frac{\gamma(t)}{2 \beta(t)}}, \\
& A_{0}=A_{0}, \\
& A_{1}=0, \\
& B_{1}=p-A_{0} p,
\end{aligned}
$$

$$
\begin{aligned}
& p=p, \\
& \mu=\mu \text {, } \\
& c(t)=\frac{1}{t} \\
& \cdot \int\left(\alpha(t)-(\rho+1) \sqrt{\frac{\gamma(t) \beta(t)}{2}}\right) d t \\
& +C_{1} \text {, } \\
& u(\xi)=A_{0}+B_{1}\left[p+\tan \left(\frac{\Phi(\xi)}{2}\right)\right]^{-1}
\end{aligned}
$$


By using (50), Families 1, 2, 7, and 14 can be written, respectively, as

$$
\begin{aligned}
& u_{33}(\xi)=A_{0}+\left(p-A_{0} p\right) \\
& {\left[p-\frac{p(\rho-1)}{2\left(\rho-A_{0}\right)}+\frac{p(\rho-1)}{2\left(\rho-A_{0}\right)} i \tan \left(\frac{\rho-1}{2 \mu} \sqrt{-\frac{\gamma(t)}{2 \beta(t)}}(\xi+C)\right)\right]^{-1},} \\
& u_{34}(\xi)=A_{0}+\left(p-A_{0} p\right) \\
& {\left[p+\frac{p(\rho-1)}{2\left(\rho-A_{0}\right)}-\frac{p(\rho-1)}{2\left(\rho-A_{0}\right)} \tanh \left(\frac{\rho-1}{2 \mu} \sqrt{\frac{\gamma(t)}{2 \beta(t)}}(\xi+C)\right)\right]^{-1},} \\
& u_{35}(\xi)=A_{0}+\left(p-A_{0} p\right) \\
& \cdot\left[p+\tan \left(\frac{1}{2} \arctan \left[\frac{2 e^{((\rho-1) / \mu) \sqrt{\gamma(t) / 2 \beta(t)}(\xi+C)}}{e^{(2(\rho-1) / \mu) \sqrt{\gamma(t) / 2 \beta(t)}(\xi+C)}+1}, \frac{e^{(2(\rho-1) / \mu) \sqrt{\gamma(t) / 2 \beta(t)}(\xi+C)}-1}{e^{(2(\rho-1) / \mu) \sqrt{\gamma(t) / 2 \beta(t)}(\xi+C)}+1}\right]\right)\right]^{-1}, \\
& u_{36}(\xi)=A_{0}+\left(p-A_{0} p\right) \\
& \cdot\left[p-\left[\frac{((\rho-1) / \mu) \sqrt{\gamma(t) / 2 \beta(t)} e^{((\rho-1) / \mu) \sqrt{\gamma(t) / 2 \beta(t)}(\xi+C)}}{\left(\left(\rho-A_{0}\right) / p \mu\right) \sqrt{\gamma(t) / 2 \beta(t)} e^{((\rho-1) / \mu) \sqrt{\gamma(t) / 2 \beta(t)}(\xi+C)}-1}\right]\right]^{-1},
\end{aligned}
$$

where $\xi=\mu\left(x-\int(\alpha(t)-(\rho+1) \sqrt{\gamma(t) \beta(t) / 2}) d t+C_{1}\right)$.

Set 17. We have the following:

$$
\begin{aligned}
& p=p, \\
& a=\frac{\rho}{\mu} \sqrt{\frac{\gamma(t)}{2 \beta(t)}}, \\
& b=-\frac{A_{0}}{p \mu} \sqrt{\frac{\gamma(t)}{2 \beta(t)}}, \\
& c=\frac{A_{0}}{p \mu} \sqrt{\frac{\gamma(t)}{2 \beta(t)}}, \\
& A_{0}=A_{0}, \\
& A_{1}=0,
\end{aligned}
$$

$$
\begin{aligned}
& B_{1}=p\left(\rho-A_{0}\right), \\
& \mu=\mu \text {, } \\
& c(t)=\frac{1}{t} \\
& \int\left(\alpha(t)-(\rho+1) \sqrt{\frac{\gamma(t) \beta(t)}{2}}\right) d t \\
& +C_{1} \text {, } \\
& u(\xi)=A_{0}+B_{1}\left[p+\tan \left(\frac{\Phi(\xi)}{2}\right)\right]^{-1} \text {. }
\end{aligned}
$$

By using (50), Families 1, 2, 7, and 14 can be written, respectively, as

$$
\begin{aligned}
& u_{37}(\xi)=A_{0}+p\left(\rho-A_{0}\right)\left[p-\frac{p \rho}{2 A_{0}}+\frac{p \rho}{2 A_{0}} i \tan \left(\frac{\rho}{2 \mu} \sqrt{-\frac{\gamma(t)}{2 \beta(t)}}(\xi+C)\right)\right]^{-1} \\
& u_{38}(\xi)=A_{0}+p\left(\rho-A_{0}\right)\left[p+\frac{p \rho}{2 A_{0}}-\frac{p \rho}{2 A_{0}} \tanh \left(\frac{\rho}{2 \mu} \sqrt{\frac{\gamma(t)}{2 \beta(t)}}(\xi+C)\right)\right]^{-1},
\end{aligned}
$$




$$
\begin{aligned}
& u_{39}(\xi)=A_{0}+p\left(\rho-A_{0}\right) \\
& \cdot\left[p+\tan \left(\frac{1}{2} \arctan \left[\frac{2 e^{(\rho / \mu) \sqrt{\gamma(t) / 2 \beta(t)}(\xi+C)}}{e^{(2 \rho / \mu) \sqrt{\gamma(t) / 2 \beta(t)(\xi+C)}+1}}, \frac{e^{(2 \rho / \mu) \sqrt{\gamma(t) / 2 \beta(t)}(\xi+C)}-1}{e^{(2 \rho / \mu) \sqrt{\gamma(t) / 2 \beta(t)}(\xi+C)}+1}\right]\right)\right]^{-1}, \\
& u_{40}(\xi)=A_{0}+\left(p-A_{0} p\right)\left[p-\left[\frac{(\rho / \mu) \sqrt{\gamma(t) / 2 \beta(t)} e^{(\rho / \mu) \sqrt{\gamma(t) / 2 \beta(t)}(\xi+C)}}{\left(A_{0} / p \mu\right) \sqrt{\gamma(t) / 2 \beta(t)} e^{(\rho / \mu) \sqrt{\gamma(t) / 2 \beta(t)(\xi+C)}-1}}\right]\right]^{-1},
\end{aligned}
$$

where $\xi=\mu\left(x-\int(\alpha(t)-(\rho+1) \sqrt{\gamma(t) \beta(t) / 2}) d t+C_{1}\right)$.

Set 18. We have the following:

$$
\begin{aligned}
& p=p, \\
& a=\frac{\rho-1}{\mu} \sqrt{\frac{\gamma(t)}{2 \beta(t)}}, \\
& b=-\frac{A_{0}-1}{p \mu} \sqrt{\frac{\gamma(t)}{2 \beta(t)}}, \\
& c=\frac{A_{0}-1}{p \mu} \sqrt{\frac{\gamma(t)}{2 \beta(t)}}, \\
& A_{0}=A_{0}, \\
& A_{1}=0, \\
& B_{1}=p\left(\rho-A_{0}\right),
\end{aligned}
$$

$$
\mu=\mu,
$$$$
c(t)=\frac{1}{t}
$$$$
\int\left(\alpha(t)+(\rho+1) \sqrt{\frac{\gamma(t) \beta(t)}{2}}\right) d t
$$$$
+C_{1} \text {, }
$$$$
u(\xi)=A_{0}+B_{1}\left[p+\tan \left(\frac{\Phi(\xi)}{2}\right)\right]^{-1}
$$

By using (50), Families 1, 2, 7, and 14 can be written, respectively, as

$$
\begin{aligned}
& u_{37}(\xi)=A_{0}+p\left(\rho-A_{0}\right) \\
& \cdot\left[p-\frac{p(\rho-1)}{2\left(A_{0}-1\right)}+\frac{p(\rho-1)}{2\left(A_{0}-1\right)} i \tan \left(\frac{\rho-1}{2 \mu} \sqrt{-\frac{\gamma(t)}{2 \beta(t)}}(\xi+C)\right)\right]^{-1}, \\
& u_{38}(\xi)=A_{0}+p\left(\rho-A_{0}\right) \\
& \cdot\left[p+\frac{p(\rho-1)}{2\left(A_{0}-1\right)}-\frac{p(\rho-1)}{2\left(A_{0}-1\right)} \tanh \left(\frac{\rho-1}{2 \mu} \sqrt{\frac{\gamma(t)}{2 \beta(t)}}(\xi+C)\right)\right]^{-1}, \\
& u_{39}(\xi)=A_{0}+p\left(\rho-A_{0}\right) \\
& \cdot\left[p+\tan \left(\frac { 1 } { 2 } \operatorname { a r c t a n } \left[\frac{2 e^{((\rho-1) / \mu) \sqrt{\gamma(t) / 2 \beta(t)(\xi+C)}}}{e^{(2(\rho-1) / \mu) \sqrt{\gamma(t) / 2 \beta(t)}(\xi+C)}+1}, \frac{e^{(2(\rho-1) / \mu) \sqrt{\gamma(t) / 2 \beta(t)(\xi+C)}-1}}{\left.\left.\left.e^{(2(\rho-1) / \mu) \sqrt{\gamma(t) / 2 \beta(t)(\xi+C)}+1}\right]\right)\right]^{-1}},\right.\right.\right.
\end{aligned}
$$




$$
\begin{aligned}
& u_{40}(\xi)=A_{0}+\left(p-A_{0} p\right) \\
& \quad\left[p-\left[\frac{((\rho-1) / \mu) \sqrt{\gamma(t) / 2 \beta(t)} e^{((\rho-1) / \mu) \sqrt{\gamma(t) / 2 \beta(t)(\xi+C)}}}{\left(\left(A_{0}-1\right) / p \mu\right) \sqrt{\gamma(t) / 2 \beta(t)} e^{((\rho-1) / \mu) \sqrt{\gamma(t) / 2 \beta(t)(\xi+C)}-1}}\right]\right]^{-1},
\end{aligned}
$$

where $\xi=\mu\left(x-\int(\alpha(t)+(\rho+1) \sqrt{\gamma(t) \beta(t) / 2}) d t+C_{1}\right)$.

Set 19. We have the following:

$$
\begin{aligned}
\mu & =\frac{1}{p c} \sqrt{\frac{\gamma(t)}{8 \beta(t)}}, \\
p & =p, \\
a & =2 p c, \\
b & =-c, \\
c & =c, \\
A_{0} & =\frac{1}{2}, \\
A_{1} & =0, \\
B_{1} & =\frac{1}{2} p(2 \rho-1), \\
c & (t)=\frac{1}{t} \\
& \cdot \int\left(\alpha(t)+(\rho-2) \sqrt{\frac{\gamma(t) \beta(t)}{8}}\right)^{d t} \\
& +C_{1}, \\
u(\xi) & =A_{0}+B_{1}\left[p+\tan \left(\frac{\Phi(\xi)}{2}\right)\right]^{-1} .
\end{aligned}
$$

By using (50), Families 1, 2, and 14 can be written, respectively, as

$$
\begin{gathered}
u_{41}(\xi)=\frac{1}{2}+(2 \rho-1) i \\
\cdot \cot \left(\sqrt{-p^{2} c^{2}}(\xi+C)\right), \\
u_{42}(\xi)=\frac{1}{2}-(2 \rho-1) \\
\cdot \operatorname{coth}(p c(\xi+C)), \\
u_{43}(\xi)=\frac{1}{2}+\frac{1}{2} p(2 \rho-1) \\
\cdot\left[p-\frac{2 p c e^{2 p c(\xi+C)}}{c e^{2 p c(\xi+C)}-1}\right]^{-1},
\end{gathered}
$$

where $\xi=(1 / p c) \sqrt{\gamma(t) / 8 \beta(t)}\left(x-\int(\alpha(t)+(\rho-\right.$ 2) $\left.\sqrt{\gamma(t) \beta(t) / 8)} d t+C_{1}\right)$.

Set 20. We have the following:

$$
\begin{aligned}
& \mu=\frac{1}{p c} \sqrt{\frac{\gamma(t)}{8 \beta(t)},} \\
& p=p, \\
& a=2 p c(1-\rho), \\
& b=-c, \\
& c=c, \\
& A_{0}=\frac{1}{2}, \\
& A_{1}=0, \\
& B_{1}=\frac{1}{2} p(2 \rho-1), \\
& c(t)=\frac{1}{t} \\
& \quad \cdot \int\left(\alpha(t)-(\rho+1) \sqrt{\frac{\gamma(t) \beta(t)}{8}}\right) d t \\
& \quad+C_{1}, \\
& u(\xi)=A_{0}+B_{1}\left[p+\tan \left(\frac{\Phi(\xi)}{2}\right)\right]^{-1} .
\end{aligned}
$$

By using (50), Families 1, 2, and 14 can be written, respectively, as

$$
\begin{aligned}
& u_{44}(\xi)=\frac{1}{2}+\frac{1}{2} p(2 \rho-1)[p \rho \\
& +p(1-\rho) i \\
& \left.\cdot \tan \left((1-\rho) \sqrt{-p^{2} c^{2}}(\xi+C)\right)\right]^{-1}, \\
& u_{45}(\xi)=\frac{1}{2}+\frac{1}{2}(2 \rho-1)[\rho-(1-\rho) \\
& \cdot \tanh (p c(1-\rho)(\xi+C))]^{-1},
\end{aligned}
$$




$$
\begin{gathered}
u_{46}(\xi)=\frac{1}{2}+\frac{1}{2}(2 \rho-1)[1 \\
\left.-\frac{2 c e^{2 p c(\xi+C)}}{c e^{2 p c(\xi+C)}-1}\right]^{-1},
\end{gathered}
$$

where $\xi=(1 / p c) \sqrt{\gamma(t) / 8 \beta(t)}\left(x-\int(\alpha(t)-(\rho+\right.$ 1) $\left.\sqrt{\gamma(t) \beta(t) / 8}) d t+C_{1}\right)$.

Set 21. We have the following:

$$
\begin{aligned}
& \mu=\frac{\rho}{p c} \sqrt{\frac{\gamma(t)}{8 \beta(t)}}, \\
& p=p, \\
& a=\frac{2 p c}{\rho}, \\
& b=-c, \\
& c=c, \\
& A_{0}=\frac{\rho}{2}, \\
& A_{1}=0, \\
& B_{1}=-\frac{1}{2} p(\rho-2), \\
& c(t)=\frac{1}{t} \int(\alpha(t) \\
& \left.\quad+(2 \rho-1) \sqrt{\frac{\gamma(t) \beta(t)}{8}}\right) d t \\
& \quad+C_{1}, \\
& u(\xi)=A_{0}+B_{1}\left[p+\tan \left(\frac{\Phi(\xi)}{2}\right)\right]^{-1} .
\end{aligned}
$$

By using (50), Families 1, 2, and 14 can be written, respectively, as

$$
\begin{array}{cc}
u_{47}(\xi)=\frac{\rho}{2}-\frac{1}{2} p(\rho-2)\left[p-\frac{p}{\rho}\right. & \cdot \int\left(\alpha(t)-(\rho+1) \sqrt{\frac{\gamma(t) \beta(t)}{8}}\right) d t \\
\left.+\frac{p}{\rho} i \tan \left(\frac{\sqrt{-p^{2} c^{2}}}{\rho}(\xi+C)\right)\right]^{-1}, & +C_{1}, \\
& u(\xi)=A_{0}+B_{1}\left[p+\tan \left(\frac{\Phi(\xi)}{2}\right)\right]^{-1} .
\end{array}
$$

$$
\begin{array}{r}
u_{48}(\xi)=\frac{\rho}{2}-\frac{1}{2}(\rho-2)\left[1-\frac{1}{\rho}\right. \\
\left.-\frac{1}{\rho} \tanh \left(\frac{p c}{\rho}(\xi+C)\right)\right]^{-1},
\end{array}
$$$$
u_{49}(\xi)=\frac{\rho}{2}-\frac{1}{2}(\rho-2)[1
$$$$
\left.-\frac{(2 c / \rho) e^{(2 p c / \rho)(\xi+C)}}{c e^{(2 p c / \rho)(\xi+C)}-1}\right]^{-1},
$$

where $\xi=(\rho / p c) \sqrt{\gamma(t) / 8 \beta(t)}\left(x-\int(\alpha(t)-(2 \rho-\right.$ 1) $\left.\sqrt{\gamma(t) \beta(t) / 8}) d t+C_{1}\right)$.

Set 22. We have the following:

$$
\begin{aligned}
& \mu=\frac{\rho}{p c} \sqrt{\frac{\gamma(t)}{8 \beta(t)}}, \\
& p=p, \\
& a=\frac{2 p c(\rho-1)}{\rho}, \\
& b=-c, \\
& c=c, \\
& A_{0}=\frac{\rho}{2}, \\
& A_{1}=0, \\
& B_{1}=-\frac{1}{2} p(\rho-2), \\
& c(t)=\frac{1}{t}
\end{aligned}
$$


By using (50), Families 1, 2, and 14 can be written, respectively, as

$$
\begin{aligned}
& u_{50}(\xi)=\frac{\rho}{2}-\frac{1}{2} p(\rho-2)\left[p-\frac{p(\rho-1)}{\rho}\right. \\
& +\frac{p(\rho-1)}{\rho} i \\
& \left.\cdot \tan \left(\frac{(\rho-1) \sqrt{-p^{2} c^{2}}}{\rho}(\xi+C)\right)\right]^{-1}, \\
& u_{51}(\xi)=\frac{\rho}{2}-\frac{1}{2} p(\rho-2)\left[p-\frac{p(\rho-1)}{\rho}\right. \\
& -\frac{p(\rho-1)}{\rho} \\
& \left.\cdot \tanh \left((\rho-1) \frac{p c}{\rho}(\xi+C)\right)\right]^{-1}, \\
& u_{52}(\xi)=\frac{\rho}{2}-\frac{1}{2} p(\rho-2)[p \\
& \left.-\frac{(2 p c(\rho-1) / \rho) e^{(2 p c(\rho-1) / \rho)(\xi+C)}}{c e^{(2 p c(\rho-1) / \rho)(\xi+C)}-1}\right]^{-1},
\end{aligned}
$$

where $\xi=(\rho / p c) \sqrt{\gamma(t) / 8 \beta(t)}\left(x-\int(\alpha(t)-(\rho+\right.$ 1) $\left.\sqrt{\gamma(t) \beta(t) / 8}) d t+C_{1}\right)$.

Set 23. We have the following:

$$
\begin{aligned}
& \mu=\frac{\rho}{p c} \sqrt{\frac{\gamma(t)}{8 \beta(t)}}, \\
& p=p, \\
& a=\frac{2 p c(\rho-1)}{\rho}, \\
& b=-c, \\
& c=c, \\
& A_{0}=\frac{\rho}{2}, \\
& A_{1}=0, \\
& B_{1}=-\frac{1}{2} p(\rho-2),
\end{aligned}
$$

$$
\begin{aligned}
& c(t)=\frac{1}{t} \\
& \quad \int\left(\alpha(t)-(\rho+1) \sqrt{\frac{\gamma(t) \beta(t)}{8}}\right) d t \\
& +C_{1}, \\
& u(\xi)=A_{0}+B_{1}\left[p+\tan \left(\frac{\Phi(\xi)}{2}\right)\right]^{-1} .
\end{aligned}
$$

By using (50), Families 1, 2, and 14 can be written, respectively, as

$$
\begin{aligned}
& u_{50}(\xi)=\frac{\rho}{2}-\frac{1}{2} p(\rho-2)\left[p-\frac{p(\rho-1)}{\rho}\right. \\
& +\frac{p(\rho-1)}{\rho} i \\
& \left.\cdot \tan \left(\frac{(\rho-1) \sqrt{-p^{2} c^{2}}}{\rho}(\xi+C)\right)\right]^{-1}, \\
& u_{51}(\xi)=\frac{\rho}{2}-\frac{1}{2} p(\rho-2)\left[p-\frac{p(\rho-1)}{\rho}\right. \\
& -\frac{p(\rho-1)}{\rho}[
\end{aligned}
$$

$$
\left.\cdot \tanh \left((\rho-1) \frac{p c}{\rho}(\xi+C)\right)\right]^{-1}
$$

$$
\begin{aligned}
& u_{52}(\xi)=\frac{\rho}{2}-\frac{1}{2} p(\rho-2)[p \\
& \left.-\frac{(2 p c(\rho-1) / \rho) e^{(2 p c(\rho-1) / \rho)(\xi+C)}}{c e^{(2 p c(\rho-1) / \rho)(\xi+C)}-1}\right]^{-1},
\end{aligned}
$$

where $\xi=(\rho / p c) \sqrt{\gamma(t) / 8 \beta(t)}\left(x-\int(\alpha(t)-(\rho+\right.$ 1) $\left.\sqrt{\gamma(t) \beta(t) / 8} d t+C_{1}\right)$.

Set 24. We have the following:

$$
\begin{aligned}
& \mu=\frac{1}{a} \sqrt{\frac{\gamma(t)}{2 \beta(t)}}, \\
& p=p, \\
& a=a,
\end{aligned}
$$




$$
\begin{aligned}
& b=\frac{a(\rho-1)}{2 p}, \\
& c=-\frac{a(\rho-1)}{2 p}, \\
& A_{0}=\frac{1}{2}(\rho+1), \\
& A_{1}=0, \\
& B_{1}=-\frac{1}{2} p(\rho+1), \\
& c(t)=\frac{1}{t} \int(\alpha(t) \\
& \left.+(2 \rho-1) \sqrt{\frac{\gamma(t) \beta(t)}{2}}\right) d t \\
& +C_{1}, \\
& u(\xi)=A_{0}+B_{1}\left[p+\tan \left(\frac{\Phi(\xi)}{2}\right)\right]^{-1}
\end{aligned}
$$

By using (50), Families 1, 2, and 14 can be written, respectively, as

$$
\begin{aligned}
& u_{53}(\xi)=\frac{1}{2}(\rho+1)-\frac{1}{2} p(\rho+1)[p \\
& +\frac{p}{\rho-1} \\
& \left.-\frac{p}{\rho-1} i \tan \left(\frac{\sqrt{-a^{2}}}{2}(\xi+C)\right)\right]^{-1}, \\
& u_{54}(\xi)=\frac{1}{2}(\rho+1)-\frac{1}{2}(\rho+1)[1 \\
& \left.+\frac{1}{\rho-1}+\frac{1}{\rho-1} \tanh \left(\frac{a}{2}(\xi+C)\right)\right]^{-1}, \\
& u_{55}(\xi)=\frac{1}{2}(\rho+1)-\frac{1}{2} p(\rho+1)[p \\
& \left.+\frac{a e^{a(\xi+C)}}{(a(\rho-1) / 2 p) e^{a(\xi+C)}+1}\right]^{-1},
\end{aligned}
$$

where $\xi=(1 / a) \sqrt{\gamma(t) / 2 \beta(t)}\left(x-\int(\alpha(t)+(2 \rho-\right.$ 1) $\left.\sqrt{\gamma(t) \beta(t) / 2}) d t+C_{1}\right)$.
Set 25. We have the following:

$$
\begin{aligned}
& \mu=\frac{\rho}{a} \sqrt{\frac{\gamma(t)}{2 \beta(t)}}, \\
& p=p, \\
& a=a, \\
& b=-\frac{a(\rho-1)}{2 p \rho} \\
& c=\frac{a(\rho-1)}{2 p \rho} \\
& A_{0}=\frac{1}{2}(\rho+1) \text {, } \\
& A_{1}=0 \text {, } \\
& B_{1}=-\frac{1}{2} p(\rho+1), \\
& c(t)=\frac{1}{t} \\
& \int\left(\alpha(t)-(\rho-2) \sqrt{\frac{\gamma(t) \beta(t)}{2}}\right) d t \\
& +C_{1} \text {, } \\
& u(\xi)=A_{0}+B_{1}\left[p+\tan \left(\frac{\Phi(\xi)}{2}\right)\right]^{-1}
\end{aligned}
$$

By using (50), Families 1, 2, and 14 can be written, respectively, as

$$
\begin{aligned}
& u_{56}(\xi)=\frac{1}{2}(\rho+1)-\frac{1}{2} p(\rho+1)[p \\
& +\frac{p \rho}{\rho-1} \\
& \left.-\frac{p \rho}{\rho-1} i \tan \left(\frac{\sqrt{-a^{2}}}{2}(\xi+C)\right)\right]^{-1}, \\
& u_{57}(\xi)=\frac{1}{2}(\rho+1)-\frac{1}{2} p(\rho+1)[p \\
& \left.+\frac{p \rho}{\rho-1}+\frac{p \rho}{\rho-1} \tanh \left(\frac{a}{2}(\xi+C)\right)\right]^{-1},
\end{aligned}
$$




$$
\begin{gathered}
u_{58}(\xi)=\frac{1}{2}(\rho+1)-\frac{1}{2} p(\rho+1)[p \\
\left.-\frac{a e^{a(\xi+C)}}{(a(\rho-1) / 2 p \rho) e^{a(\xi+C)}-1}\right]^{-1},
\end{gathered}
$$

where $\xi=(\rho / a) \sqrt{\gamma(t) / 2 \beta(t)}\left(x-\int(\alpha(t)-(\rho-\right.$ 2) $\left.\sqrt{\gamma(t) \beta(t) / 2)} d t+C_{1}\right)$.

Set 26. We have the following:

$$
\begin{aligned}
& \mu=\frac{1}{a} \sqrt{\frac{\gamma(t)}{2 \beta(t)}} \\
& p=p, \\
& a=a, \\
& b=c \\
& c=c \\
& A_{0}=0 \\
& A_{1}=0, \\
& B_{1}=p-\frac{c}{a}, \\
& c(t)=\frac{1}{t} \int(\alpha(t) \\
& \left.\quad-(2 \rho-1) \sqrt{\frac{\gamma(t) \beta(t)}{2}}\right) d t \\
& \quad+C_{1}, \\
& u(\xi)=B_{1}\left[p+\tan \left(\frac{\Phi(\xi)}{2}\right)\right]^{-1},
\end{aligned}
$$

where $a, b$, and $c$ are arbitrary constants. By using (77), Families 7 and 9 can be written, respectively, as

$$
\begin{aligned}
& u_{59}(\xi)=\left[1+\frac{1}{p} \tan \left(\frac{1}{2}\right.\right. \\
& \left.\left.\cdot \arctan \left[\frac{2 e^{a(\xi+C)}}{e^{2 a(\xi+C)}+1}, \frac{e^{2 a(\xi+C)}-1}{e^{2 a(\xi+C)}+1}\right]\right)\right]^{-1}, \\
& u_{60}(\xi)=\left[1+\frac{1}{p-1} e^{k a(\xi+C)}\right]^{-1},
\end{aligned}
$$

where $\xi=(1 / a) \sqrt{\gamma(t) / 2 \beta(t)}\left(x-\int(\alpha(t)-(2 \rho-\right.$ 1) $\left.\sqrt{\gamma(t) \beta(t) / 2}) d t+C_{1}\right)$.

Set 27. We have the following:

$$
\begin{aligned}
& \mu=\frac{\rho}{a} \sqrt{\frac{\gamma(t)}{2 \beta(t)}}, \\
& p=p \\
& a=a, \\
& b=c, \\
& c=c, \\
& A_{0}=0, \\
& A_{1}=0, \\
& B_{1}=\left(p-\frac{c}{\mathrm{a}}\right) \rho, \\
& c(t)=\frac{1}{t} \\
& \quad \cdot \int\left(\alpha(t)+(\rho-2) \sqrt{\frac{\gamma(t) \beta(t)}{2}}\right) d t \\
& \quad+C_{1}, \\
& u(\xi)=B_{1}\left[p+\tan \left(\frac{\Phi(\xi)}{2}\right)\right]^{-1}, \\
& \quad\left(\frac{1}{2}\right)
\end{aligned}
$$

where $a, b$, and $c$ are arbitrary constants. By using (80), Families 7 and 9 can be written, respectively, as

$$
\begin{aligned}
& u_{61}(\xi)=\rho\left[1+\frac{1}{p} \tan \left(\frac{1}{2}\right.\right. \\
& \left.\left.\cdot \arctan \left[\frac{2 e^{a(\xi+C)}}{e^{2 a(\xi+C)}+1}, \frac{e^{2 a(\xi+C)}-1}{e^{2 a(\xi+C)}+1}\right]\right)\right]^{-1}, \\
& u_{62}(\xi)=\rho\left[+\frac{1}{p-1} e^{k a(\xi+C)}\right]^{-1},
\end{aligned}
$$

where $\xi=(\rho / a) \sqrt{\gamma(t) / 2 \beta(t)}\left(x-\int(\alpha(t)+(\rho-\right.$ 2) $\left.\sqrt{\gamma(t) \beta(t) / 2}) d t+C_{1}\right)$. 
Set 28. We have the following:

$$
\begin{aligned}
& \mu=\frac{1}{a} \sqrt{\frac{\gamma(t)}{2 \beta(t)}} \\
& p=p \\
& a=a, \\
& b=c, \\
& c=c, \\
& A_{0}=1, \\
& A_{1}=0, \\
& B_{1}=\left(-p+\frac{c}{a}\right), \\
& +(\xi)=\frac{1}{t} \int(\alpha(t) \\
& +(t) \\
& \left.+(2 \rho-1) \sqrt{\frac{\gamma(t) \beta(t)}{2}}\right) d t
\end{aligned}
$$

where $a, b$, and $c$ are arbitrary constants. By using (83), Families 7 and 9, respectively, can be written as

$$
\begin{aligned}
& u_{63}(\xi)=1-p\left[p+\tan \left(\frac{1}{2}\right.\right. \\
& \left.\left.\cdot \arctan \left[\frac{2 e^{a(\xi+C)}}{e^{2 a(\xi+C)}+1}, \frac{e^{2 a(\xi+C)}-1}{e^{2 a(\xi+C)}+1}\right]\right)\right]^{-1}, \\
& u_{64}(\xi)=1+(1-p)\left[p-1+e^{k a(\xi+C)}\right]^{-1},
\end{aligned}
$$

where $\xi=(1 / a) \sqrt{\gamma(t) / 2 \beta(t)}\left(x-\int(\alpha(t)+(2 \rho-\right.$ 1) $\left.\sqrt{\gamma(t) \beta(\mathrm{t}) / 2}) d t+C_{1}\right)$.

Set 29. We have the following:

$$
\begin{aligned}
& \mu=\frac{\rho-1}{a} \sqrt{\frac{\gamma(t)}{2 \beta(t)}}, \\
& p=p, \\
& a=a,
\end{aligned}
$$

$$
\begin{aligned}
& b=c \\
& c=c \\
& A_{0}=1, \\
& A_{1}=0 \\
& B_{1}=\left(p-\frac{c}{a}\right)(\rho-1), \\
& \left.c(t)=\frac{1}{t}\right)^{-}\left(\alpha(t)+(\rho+1) \sqrt{\frac{\gamma(t) \beta(t)}{2}}\right)^{-1} d t \\
& \quad+C_{1}, \\
& u(\xi)=A_{0}+B_{1}\left[p+\tan \left(\frac{\Phi(\xi)}{2}\right)\right. \\
& \quad \int
\end{aligned}
$$

where $a, b$, and $c$ are arbitrary constants. By using (86), Families 7 and 9 can be written, respectively, as

$$
\begin{gathered}
u_{65}(\xi)=1+(\rho-1)\left[1+\frac{1}{p} \tan \left(\frac{1}{2}\right.\right. \\
\left.\left.\cdot \arctan \left[\frac{2 e^{a(\xi+C)}}{e^{2 a(\xi+C)}+1}, \frac{e^{2 a(\xi+C)}-1}{e^{2 a(\xi+C)}+1}\right]\right)\right]^{-1}, \\
u_{66}(\xi)=1+(\rho-1)\left[1+\frac{1}{p-1} e^{k a(\xi+C)}\right]^{-1},
\end{gathered}
$$

where $\xi=((\rho-1) / a) \sqrt{\gamma(t) / 2 \beta(t)}\left(x-\int(\alpha(t)+(\rho+\right.$ 1) $\left.\sqrt{\gamma(t) \beta(t) / 2}) d t+C_{1}\right)$.

Set 30. We have the following:

$$
\begin{aligned}
& \mu=\frac{\rho}{a} \sqrt{\frac{\gamma(t)}{2 \beta(t)}}, \\
& p=p, \\
& a=a, \\
& b=c, \\
& c=c,
\end{aligned}
$$




$$
\begin{aligned}
& A_{0}=\rho \\
& A_{1}=0 \\
& B_{1}=\left(-p+\frac{c}{a}\right) \rho, \\
& c(t)=\frac{1}{t} \\
& \left.\cdot \int \alpha(t)-(\rho-2) \sqrt{\frac{\gamma(t) \beta(t)}{2}}\right)^{d t} \\
& +C_{1}, \\
& u(\xi)=A_{0}+B_{1}\left[p+\tan \left(\frac{\Phi(\xi)}{2}\right)\right]^{-1},
\end{aligned}
$$

$$
\begin{aligned}
& c(t)=\frac{1}{t} \\
& \quad \cdot\left(\alpha(t)-(\rho+1) \sqrt{\frac{\gamma(t) \beta(t)}{2}}\right) d t \\
& +C_{1}, \\
& u(\xi)=A_{0}+B_{1}\left[p+\tan \left(\frac{\Phi(\xi)}{2}\right)\right]^{-1},
\end{aligned}
$$

where $a, b$, and $c$ are arbitrary constants. By using (92), Families 7 and 9 can be written, respectively, as

$$
u_{69}(\xi)=\rho-(\rho-1)\left[1+\frac{1}{p} \tan \left(\frac{1}{2}\right.\right.
$$

where $a, b$, and $c$ are arbitrary constants. By using (89), Families 7 and 9 can be written, respectively, as

$$
\begin{aligned}
& \cdot \arctan \left[\frac{2 e^{a(\xi+C)}}{e^{2 a(\xi+C)}+1},\right. \\
& \left.\left.\left.\frac{e^{2 a(\xi+C)}-1}{e^{2 a(\xi+C)}+1}\right]\right)\right]^{-1}, \\
& u_{70}(\xi)=\rho+\left(\frac{c}{a}-p\right)(\rho-1)[p-1 \\
& \left.+e^{k a(\xi+C)}\right]^{-1},
\end{aligned}
$$

where $\xi=((\rho-1) / a) \sqrt{\gamma(t) / 2 \beta(t)}\left(x-\int(\alpha(t)-(\rho+\right.$ 1) $\left.\sqrt{\gamma(t) \beta(t) / 2}) d t+C_{1}\right)$.

Set 32. We have the following:

2) $\left.\sqrt{\gamma(t) \beta(t) / 2}) d t+C_{1}\right)$.

Set 31. We have the following:

$$
\begin{aligned}
& \mu=\frac{\rho-1}{a} \sqrt{\frac{\gamma(t)}{2 \beta(t)}}, \\
& p=p, \\
& a=a, \\
& b=c, \\
& c=c, \\
& A_{0}=\rho, \\
& A_{1}=0, \\
& B_{1}=\left(-p+\frac{c}{a}\right)(\rho-1),
\end{aligned}
$$

$$
\begin{aligned}
& \mu=\frac{1}{p c} \sqrt{\frac{\gamma(t)}{32 \beta(t)}}, \\
& p=p, \\
& a=2 p c, \\
& b=-c, \\
& c=c, \\
& A_{0}=\frac{1}{2}, \\
& A_{1}=-\frac{1}{4 p}, \\
& B_{1}=-\frac{p}{4},
\end{aligned}
$$




$$
\begin{aligned}
& c(t)=\frac{1}{t} \int(\alpha(t) \\
& \left.+(2 \rho-1) \sqrt{\frac{\gamma(t) \beta(t)}{2}}\right) d t \\
& +C_{1}, \\
& u(\xi)=A_{0}+A_{1}\left[p+\tan \left(\frac{\Phi(\xi)}{2}\right)\right] \\
& +B_{1}\left[p+\tan \left(\frac{\Phi(\xi)}{2}\right)\right]^{-1},
\end{aligned}
$$

where $c$ is an arbitrary constant. By using (95), Families 1, 2, and 14 can be written, respectively, as

$$
\begin{aligned}
& u_{71}(\xi)=\frac{1}{2}-\frac{1}{4} \\
& \cdot i\left[\tan \left(\sqrt{-p^{2} c^{2}}(\xi+C)\right)\right. \\
& \left.-\cot \left(\sqrt{-p^{2} c^{2}}(\xi+C)\right)\right], \\
& u_{72}(\xi)=\frac{1}{2}+\frac{1}{4}\left[\tanh \left(\sqrt{p^{2} c^{2}}(\xi+C)\right)\right. \\
& \left.+\operatorname{coth}\left(\sqrt{p^{2} c^{2}}(\xi+C)\right)\right], \\
& u_{73}(\xi)=\frac{1}{2}-\frac{1}{4}\left[1-\frac{2 c e^{2 p c(\xi+C)}}{c e^{2 p c(\xi+C)}-1}\right] \\
& -\frac{1}{4}\left[1-\frac{2 c e^{2 p c(\xi+C)}}{c e^{2 p c(\xi+C)}-1}\right]^{-1},
\end{aligned}
$$

where $\xi=(1 / p c) \sqrt{\gamma(t) / 32 \beta(t)}\left(x-\int(\alpha(t)+(2 \rho-\right.$ 1) $\left.\sqrt{\gamma(t) \beta(t) / 2}) d t+C_{1}\right)$.

Set 33. We have the following:

$$
\begin{aligned}
& \mu=\frac{\rho}{p c} \sqrt{\frac{\gamma(t)}{32 \beta(t)}}, \\
& p=p, \\
& a=2 p c, \\
& b=-c, \\
& c=c,
\end{aligned}
$$

$$
\begin{aligned}
& A_{0}=\frac{\rho}{2}, \\
& A_{1}=-\frac{\rho}{4 p}, \\
& B_{1}=-\frac{p \rho}{4}, \\
& \begin{aligned}
c(t) & =\frac{1}{t} \\
\cdot \int & \left(\alpha(t)-(\rho-2) \sqrt{\frac{\gamma(t) \beta(t)}{2}}\right) d t \\
& +C_{1},
\end{aligned}
\end{aligned}
$$

$$
\begin{gathered}
u(\xi)=A_{0}+A_{1}\left[p+\tan \left(\frac{\Phi(\xi)}{2}\right)\right] \\
+B_{1}\left[p+\tan \left(\frac{\Phi(\xi)}{2}\right)\right]^{-1},
\end{gathered}
$$

where $c$ is an arbitrary constant. By using (99), Families 1, 2, and 14 can be written, respectively, as

$$
\begin{gathered}
u_{74}(\xi)=\frac{\rho}{2}-\frac{\rho}{4} \\
\cdot i\left[\tan \left(\sqrt{-p^{2} c^{2}}(\xi+C)\right)\right. \\
\left.-\cot \left(\sqrt{-p^{2} c^{2}}(\xi+C)\right)\right], \\
u_{75}(\xi)=\frac{\rho}{2} \\
+\frac{\rho}{4}\left[\tanh \left(\sqrt{p^{2} c^{2}}(\xi+C)\right)\right. \\
\left.+\operatorname{coth}\left(\sqrt{p^{2} c^{2}}(\xi+C)\right)\right],
\end{gathered}
$$

$$
\begin{gathered}
u_{76}(\xi)=\frac{\rho}{2}-\frac{\rho}{4}\left[1-\frac{2 c e^{2 p c(\xi+C)}}{c e^{2 p c(\xi+C)}-1}\right] \\
-\frac{\rho}{4}\left[1-\frac{2 c e^{2 p c(\xi+C)}}{c e^{2 p c(\xi+C)}-1}\right]^{-1},
\end{gathered}
$$

where $\xi=(\rho / p c) \sqrt{\gamma(t) / 32 \beta(t)}\left(x-\int(\alpha(t)-(\rho-\right.$ 2) $\left.\sqrt{\gamma(t) \beta(t) / 2}) d t+C_{1}\right)$. 
Set 34. We have the following:

$$
\begin{aligned}
& \mu=\frac{1}{p c} \sqrt{\frac{\gamma(t)}{32 \beta(t)}}, \\
& p=p \\
& a=2 p c, \\
& b=-c \text {, } \\
& c=c, \\
& A_{0}=\frac{1}{2} \text {, } \\
& A_{1}=\frac{1}{4 p} \text {, } \\
& B_{1}=\frac{p}{4} \text {, } \\
& c(t)=\frac{1}{t} \int(\alpha(t) \\
& \left.-(2 \rho-1) \sqrt{\frac{\gamma(t) \beta(t)}{2}}\right) d t \\
& +C_{1} \text {, } \\
& u(\xi)=A_{0}+A_{1}\left[p+\tan \left(\frac{\Phi(\xi)}{2}\right)\right] \\
& +B_{1}\left[p+\tan \left(\frac{\Phi(\xi)}{2}\right)\right]^{-1}
\end{aligned}
$$

where $c$ is an arbitrary constant. By using (102), Families 1, 2, and 14 can be written, respectively, as

$$
\begin{aligned}
& u_{77}(\xi)=\frac{1}{2}+\frac{1}{4} \\
& \cdot i\left[\tan \left(\sqrt{-p^{2} c^{2}}(\xi+C)\right)\right. \\
& \left.-\cot \left(\sqrt{-p^{2} c^{2}}(\xi+C)\right)\right], \\
& u_{78}(\xi)=\frac{1}{2} \\
& -\frac{1}{4}\left[\tanh \left(\sqrt{p^{2} c^{2}}(\xi+C)\right)\right. \\
& \left.+\operatorname{coth}\left(\sqrt{p^{2} c^{2}}(\xi+C)\right)\right]
\end{aligned}
$$

$$
\begin{gathered}
u_{79}(\xi)=\frac{1}{2}+\frac{1}{4}\left[1-\frac{2 c e^{2 p c(\xi+C)}}{c e^{2 p c(\xi+C)}-1}\right] \\
+\frac{1}{4}\left[1-\frac{2 c e^{2 p c(\xi+C)}}{c e^{2 p c(\xi+C)}-1}\right]^{-1},
\end{gathered}
$$

where $\xi=(1 / p c) \sqrt{\gamma(t) / 32 \beta(t)}\left(x-\int(\alpha(t)-(2 \rho-\right.$ 1) $\left.\sqrt{\gamma(t) \beta(t) / 2}) d t+C_{1}\right)$.

Set 35. We have the following:

$$
\begin{aligned}
& \mu=\frac{\rho-1}{p c} \sqrt{\frac{\gamma(t)}{32 \beta(t)}}, \\
& p=p \\
& a=2 p c, \\
& b=-c \text {, } \\
& c=c, \\
& A_{0}=\frac{1}{2}(\rho+1) \text {, } \\
& A_{1}=-\frac{\rho-1}{4 p}, \\
& B_{1}=-\frac{p(\rho-1)}{4}, \\
& c(t)=\frac{1}{t} \int(\alpha(t) \\
& \left.-(\rho+1) \sqrt{\frac{\gamma(t) \beta(t)}{2}}\right) d t \\
& +C_{1} \text {, } \\
& u(\xi)=A_{0}+A_{1}\left[p+\tan \left(\frac{\Phi(\xi)}{2}\right)\right] \\
& +B_{1}\left[p+\tan \left(\frac{\Phi(\xi)}{2}\right)\right]^{-1},
\end{aligned}
$$

where $c$ is an arbitrary constant. By using (105), Families 1, 2, and 14 can be written, respectively, as

$$
\begin{gathered}
u_{80}(\xi)=\frac{1}{2}(\rho+1)-\frac{\rho-1}{4} \\
\cdot i\left[\tan \left(\sqrt{-p^{2} c^{2}}(\xi+C)\right)\right. \\
\left.-\cot \left(\sqrt{-p^{2} c^{2}}(\xi+C)\right)\right],
\end{gathered}
$$




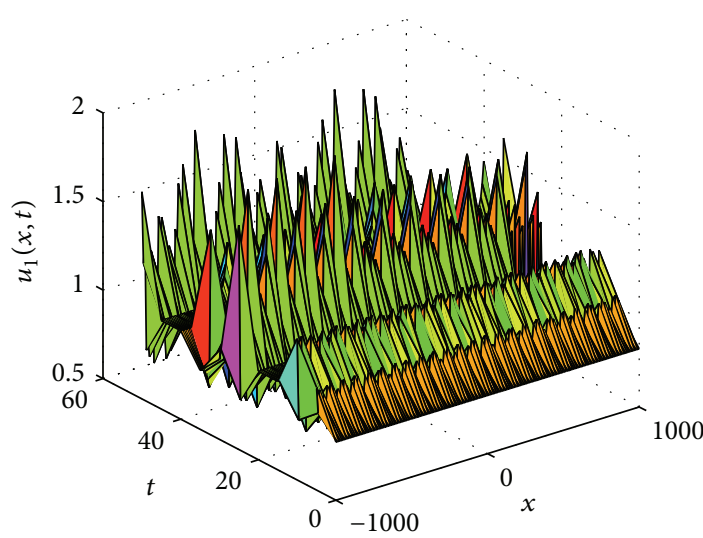

(a)

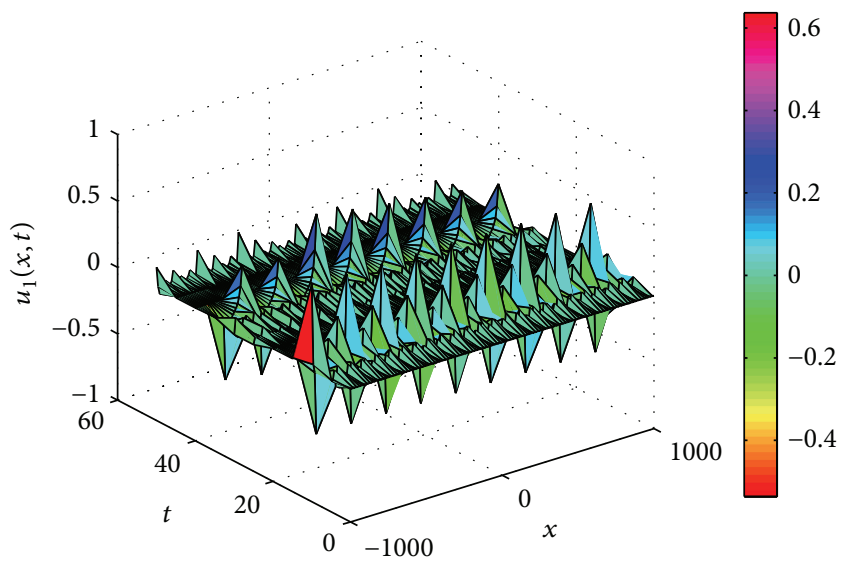

(c)

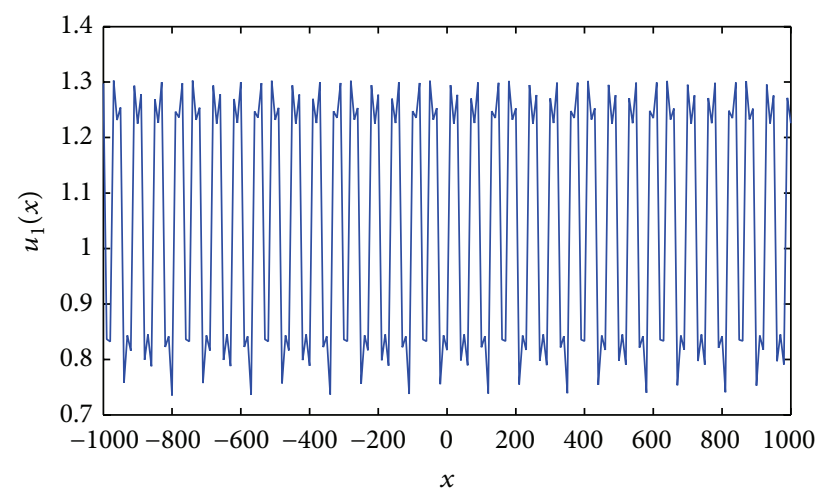

(b)

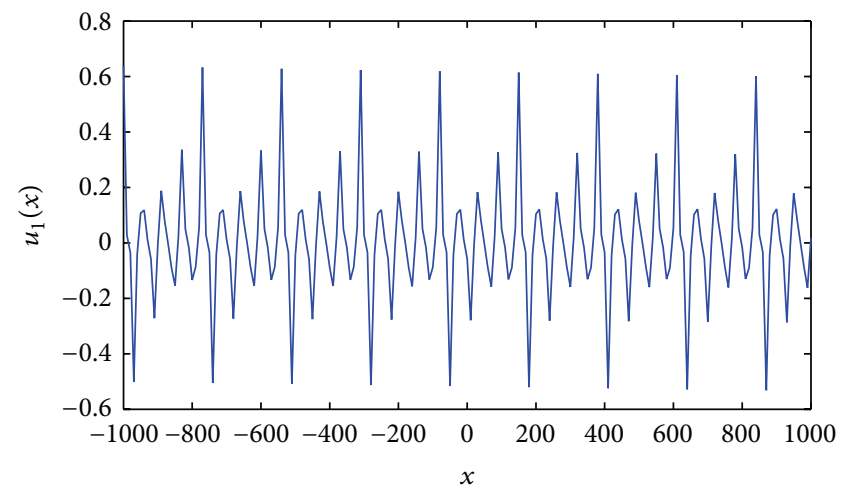

(d)

Figure 1: Graphs of ((a) and (b)) real values and ((c) and (d)) imaginary values of (14) are demonstrated at $C_{1}=2, p=2, \rho=2, a=$ 2, $\beta(t)=\cos (t), \gamma(t)=\sin (2 t)$, and $\alpha(t)=\sin (t)$ and by considering the values $((\mathrm{a})$ and $(\mathrm{c}))-1000<x<1000,0<t<50$ and $((\mathrm{b})$ and (d) $-1000<x<1000, t=10$.

$$
\begin{aligned}
& u_{81}(\xi)=\frac{1}{2}(\rho+1) \\
& +\frac{\rho-1}{4}\left[\tanh \left(\sqrt{p^{2} c^{2}}(\xi+C)\right)\right. \\
& \left.+\operatorname{coth}\left(\sqrt{p^{2} c^{2}}(\xi+C)\right)\right] \\
& u_{82}(\xi)=\frac{1}{2}(\rho+1)-\frac{\rho-1}{4}[1 \\
& \left.-\frac{2 c e^{2 p c(\xi+C)}}{c e^{2 p c(\xi+C)}-1}\right]^{-\frac{\rho-1}{4}}[1 \\
& \left.-\frac{2 c e^{2 p c(\xi+C)}}{c e^{2 p c(\xi+C)}-1}\right]^{-1},
\end{aligned}
$$

where $\xi=((\rho-1) / p c) \sqrt{\gamma(t) / 32 \beta(t)}\left(x-\int(\alpha(t)-(\rho+\right.$ 1) $\left.\sqrt{\gamma(t) \beta(t) / 2}) d t+C_{1}\right)$.
Set 36. We have the following:

$$
\begin{aligned}
& \mu=\frac{\rho}{p c} \sqrt{\frac{\gamma(t)}{32 \beta(t)}}, \\
& p=p, \\
& a=2 p c \\
& b=-c \\
& c=c \\
& A_{0}=\frac{\rho}{2}, \\
& A_{1}=\frac{\rho}{4 p}
\end{aligned}
$$




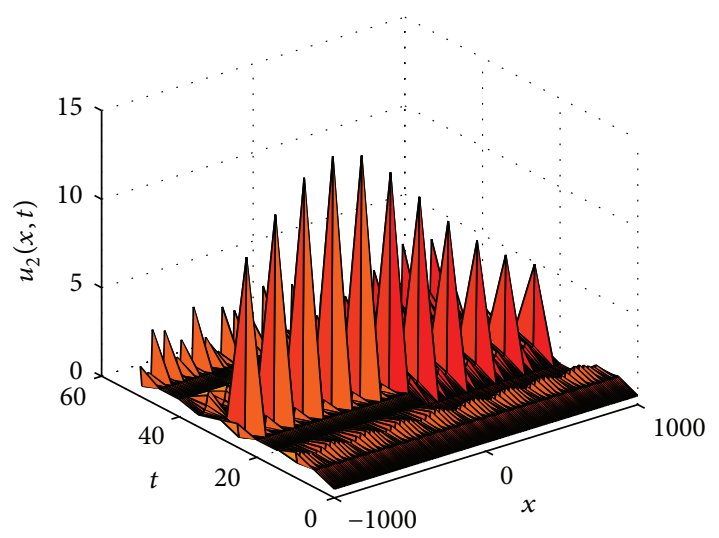

(a)

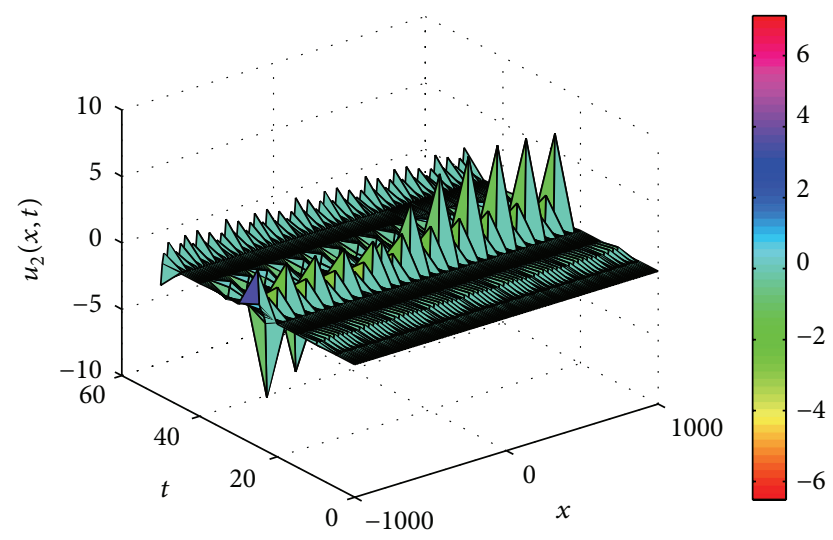

(c)

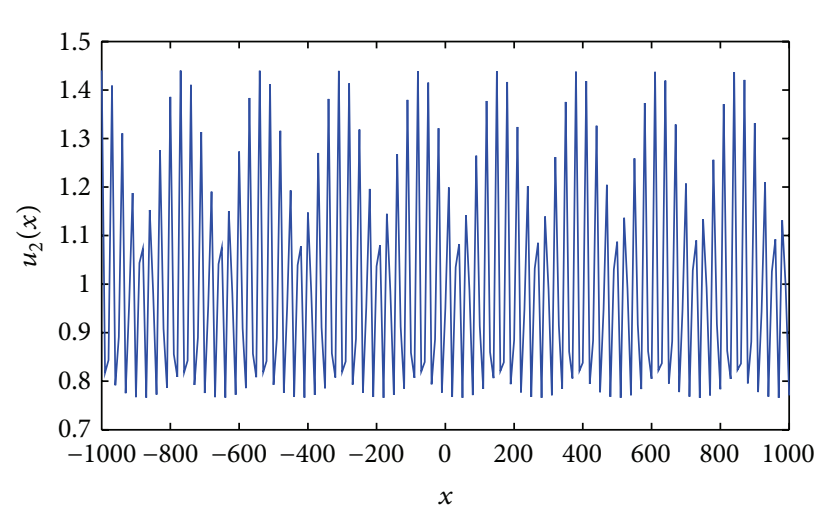

(b)

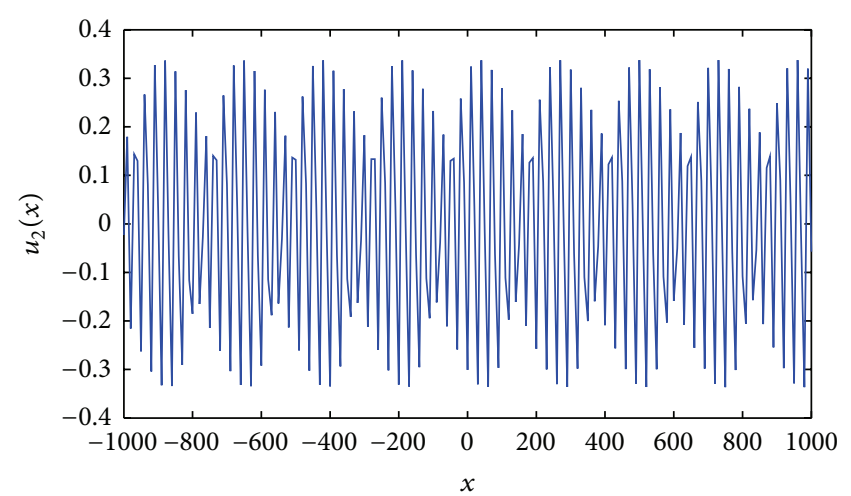

(d)

FIGURE 2: Graphs of ((a) and (b)) real values and ((c) and (d)) imaginary values of (15) are demonstrated at $C_{1}=2, p=2, k=2, \rho=2, a=$ 2, $\beta(t)=\cos (t), \gamma(t)=\sin (2 t)$, and $\alpha(t)=\sin (t)$ and by considering the values $((\mathrm{a})$ and $(\mathrm{c}))-1000<x<1000,0<t<50$ and $((\mathrm{b})$ and (d) $)-1000<x<1000, t=10$.

$$
\begin{aligned}
& c(t)=\frac{1}{t} \int(\alpha(t) \\
& \left.+(\rho-2) \sqrt{\frac{\gamma(t) \beta(t)}{2}}\right) d t \\
& +C_{1}, \\
& u(\xi)=A_{0}+A_{1}\left[p+\tan \left(\frac{\Phi(\xi)}{2}\right)\right] \\
& +B_{1}\left[p+\tan \left(\frac{\Phi(\xi)}{2}\right)\right]^{-1},
\end{aligned}
$$

where $c$ is an arbitrary constant. By using (108), Families 1, 2, and 14 can be written, respectively, as

$$
\begin{aligned}
& u_{83}(\xi)=\frac{\rho}{2}+\frac{\rho}{4} \\
& \cdot i\left[\tan \left(\sqrt{-p^{2} c^{2}}(\xi+C)\right)\right.
\end{aligned}
$$

$$
\begin{aligned}
& \left.-\cot \left(\sqrt{-p^{2} c^{2}}(\xi+C)\right)\right] \\
& u_{84}(\xi)=\frac{\rho}{2} \\
& -\frac{\rho}{4}\left[\tanh \left(\sqrt{p^{2} c^{2}}(\xi+C)\right)\right. \\
& \left.+\operatorname{coth}\left(\sqrt{p^{2} c^{2}}(\xi+C)\right)\right] \\
& u_{85}(\xi)=\frac{\rho}{2}+\frac{\rho}{4}\left[1-\frac{2 c e^{2 p c(\xi+C)}}{c e^{2 p c(\xi+C)}-1}\right] \\
& +\frac{\rho}{4}\left[1-\frac{2 c e^{2 p c(\xi+C)}}{c e^{2 p c(\xi+C)}-1}\right]^{-1},
\end{aligned}
$$

where $\xi=(\rho / p c) \sqrt{\gamma(t) / 32 \beta(t)}\left(x-\int(\alpha(t)+(\rho-\right.$ 2) $\left.\sqrt{\gamma(t) \beta(t) / 2}) d t+C_{1}\right)$. 


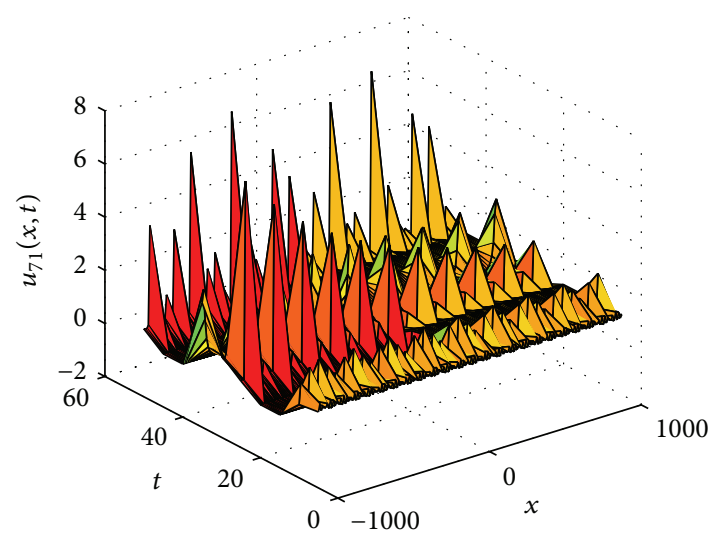

(a)

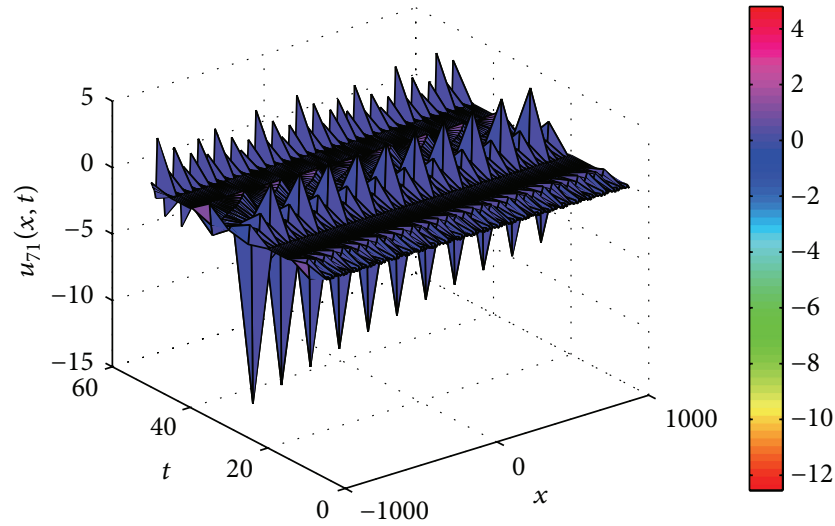

(c)

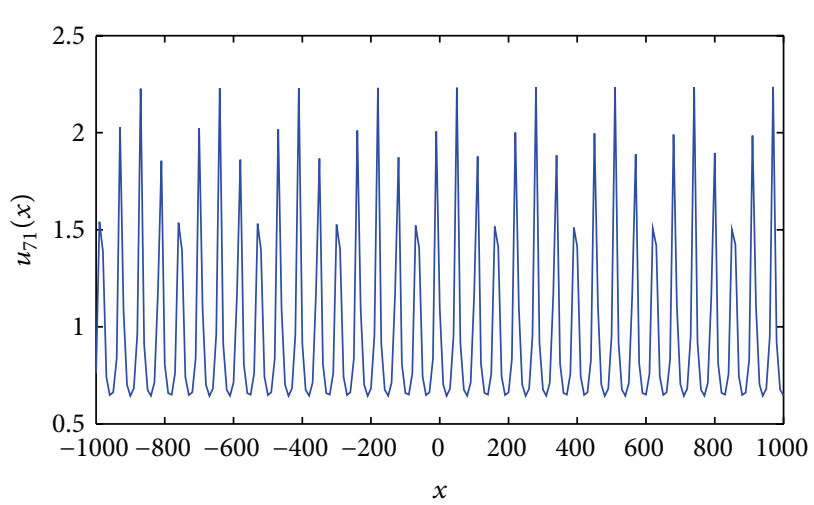

(b)

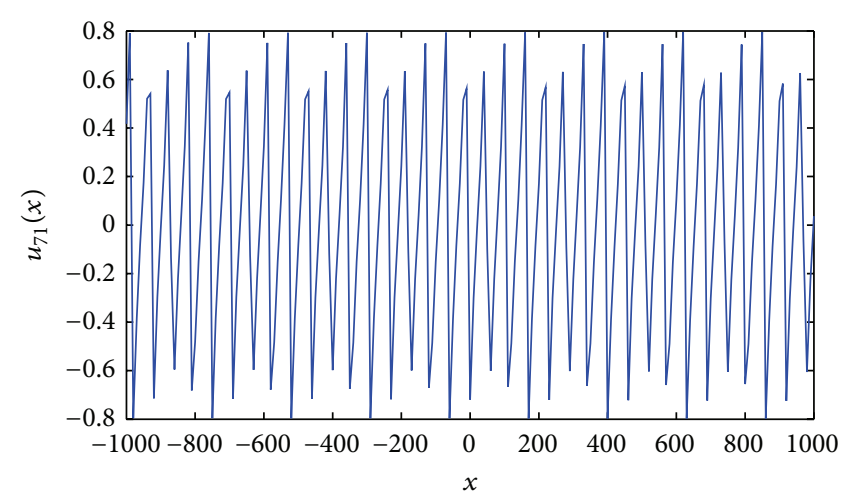

(d)

FIGURE 3: Graphs of ((a) and (b)) real values and ((c) and (d)) imaginary values of (96) are demonstrated at $C_{1}=2, p=2, k=2, \rho=2, c=$ 2, $\beta(t)=\cos (t), \gamma(t)=\sin (2 t)$, and $\alpha(t)=\sin (t)$ and by considering the values $((\mathrm{a})$ and $(\mathrm{c}))-1000<x<1000,0<t<50$ and $((\mathrm{b})$ and (d) $)-1000<x<1000, t=10$.

Set 37. We have the following:

$$
\begin{aligned}
& \mu=\frac{\rho-1}{p c} \sqrt{\frac{\gamma(t)}{32 \beta(t)}} \\
& p=p \\
& a=2 p c \\
& b=-c \\
& c=c \\
& A_{0}=\frac{1}{2}(\rho+1) \\
& A_{1}=\frac{\rho-1}{4 p} \\
& B_{1}=\frac{p(\rho-1)}{4}
\end{aligned}
$$

$$
\begin{gathered}
u(\xi)=A_{0}+A_{1}\left[p+\tan \left(\frac{\Phi(\xi)}{2}\right)\right] \\
+B_{1}\left[p+\tan \left(\frac{\Phi(\xi)}{2}\right)\right]^{-1},
\end{gathered}
$$

where $c$ is an arbitrary constant. By using (111), Families 1, 2, and 14 can be written, respectively, as

$$
\begin{gathered}
u_{86}(\xi)=\frac{1}{2}(\rho+1)+\frac{\rho-1}{4} \\
\cdot i\left[\tan \left(\sqrt{-p^{2} c^{2}}(\xi+C)\right)\right.
\end{gathered}
$$




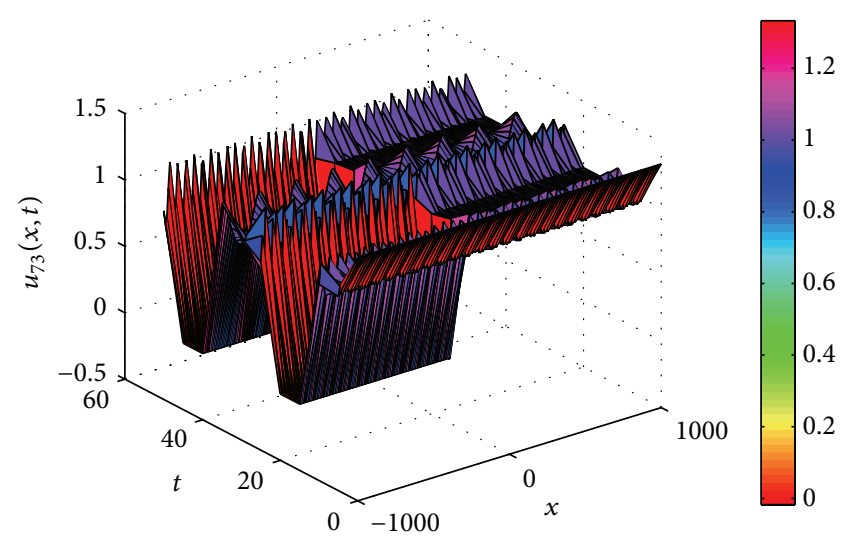

(a)

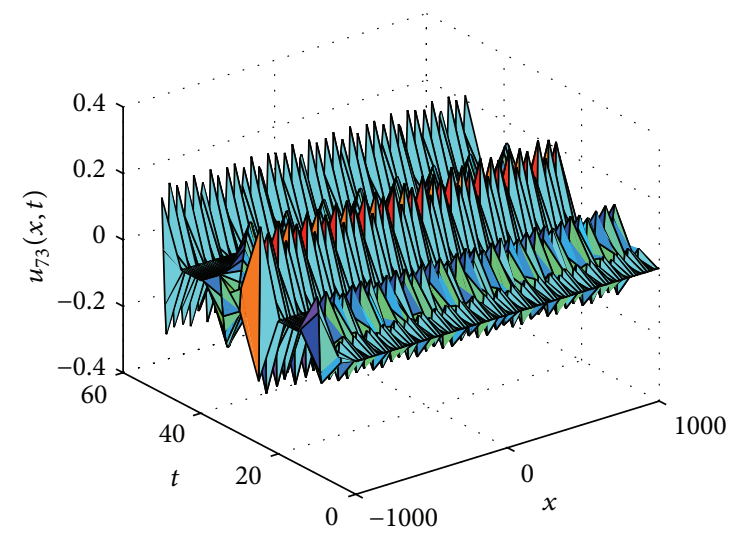

(c)

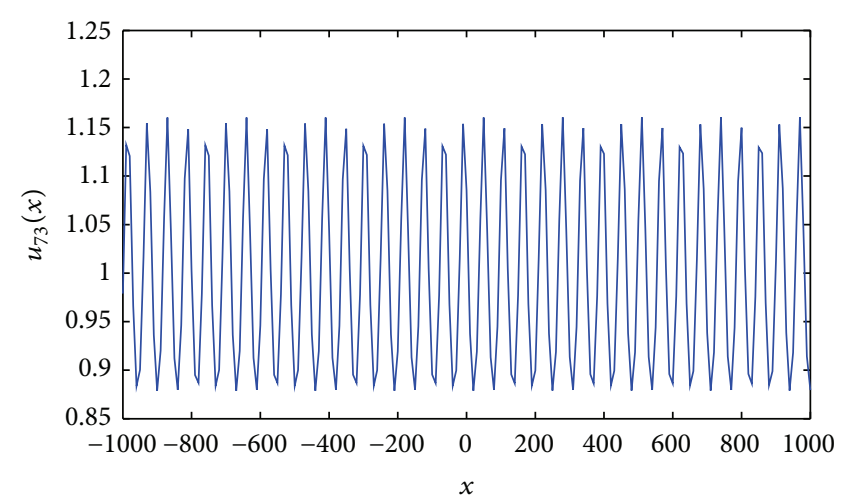

(b)

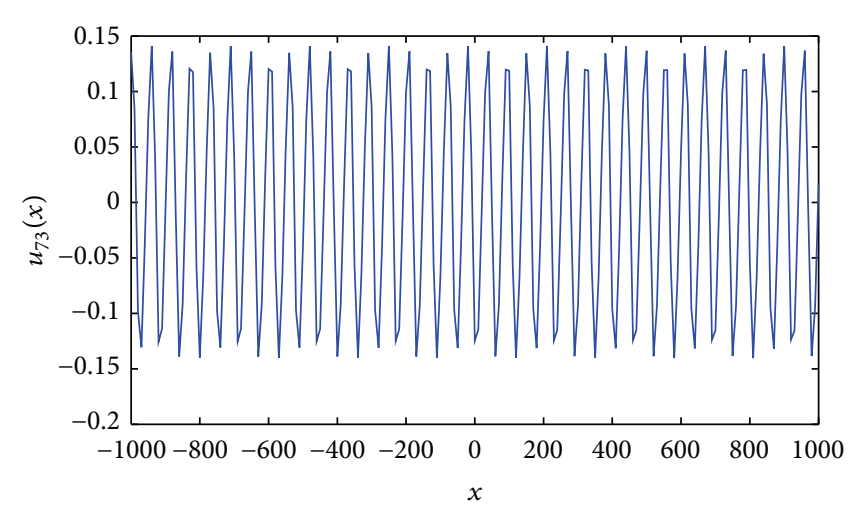

(d)

FiguRE 4: Graphs of ((a) and (b)) real values and ((c) and (d)) imaginary values of (97) are demonstrated at $C_{1}=2, p=2, k=2, \rho=2, c=$ 2, $\beta(t)=\cos (t), \gamma(t)=\sin (2 t)$, and $\alpha(t)=\sin (t)$ and by considering the values $((\mathrm{a})$ and $(\mathrm{c}))-1000<x<1000,0<t<50$ and $((\mathrm{b})$ and (d) $-1000<x<1000, t=10$.

$$
\begin{gathered}
-\cot \left(\sqrt{-p^{2} c^{2}}(\xi+C)\right), \\
u_{87}(\xi)=\frac{1}{2}(\rho+1) \\
-\frac{\rho-1}{4}\left[\tanh \left(\sqrt{p^{2} c^{2}}(\xi+C)\right)\right. \\
+\operatorname{coth}\left(\sqrt{p^{2} c^{2}}(\xi+C)\right), \\
u_{88}(\xi)=\frac{1}{2}(\rho+1)+\frac{\rho-1}{4}[1 \\
\left.-\frac{2 c e^{2 p c(\xi+C)}}{c e^{2 p c(\xi+C)}-1}\right]+\frac{\rho-1}{4}[1 \\
\left.-\frac{2 c e^{2 p c(\xi+C)}}{c e^{2 p c(\xi+C)}-1}\right]^{-1},
\end{gathered}
$$

where $\xi=((\rho-1) / p c) \sqrt{\gamma(t) / 32 \beta(t)}\left(x-\int(\alpha(t)+(\rho+\right.$ 1) $\left.\sqrt{\gamma(t) \beta(t) / 2}) d t+C_{1}\right)$.

Set 38. We have the following:

$$
\begin{aligned}
& \mu=\frac{A_{1}}{c} \sqrt{\frac{\gamma(t)}{2 \beta(t)}}, \\
& p=p \\
& a=-\frac{c(\rho-1)}{A_{1}}, \\
& b=-c \\
& c=c \\
& A_{0}=1-p A_{1}, \\
& A_{1}=A_{1}, \\
& B_{1}=0
\end{aligned}
$$




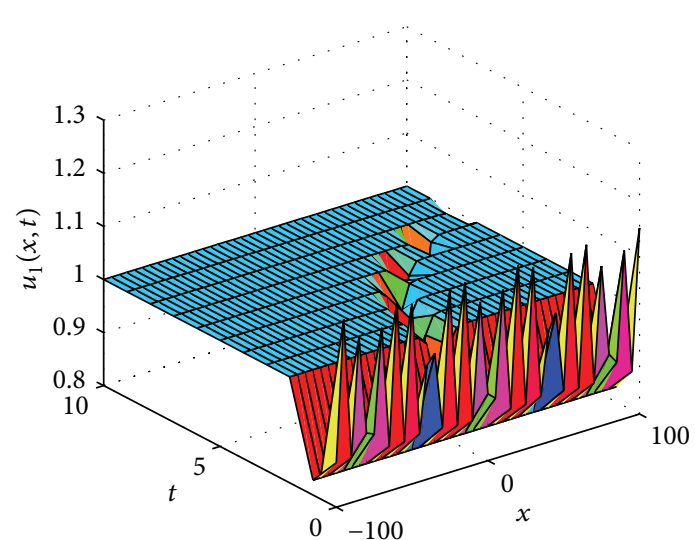

(a)

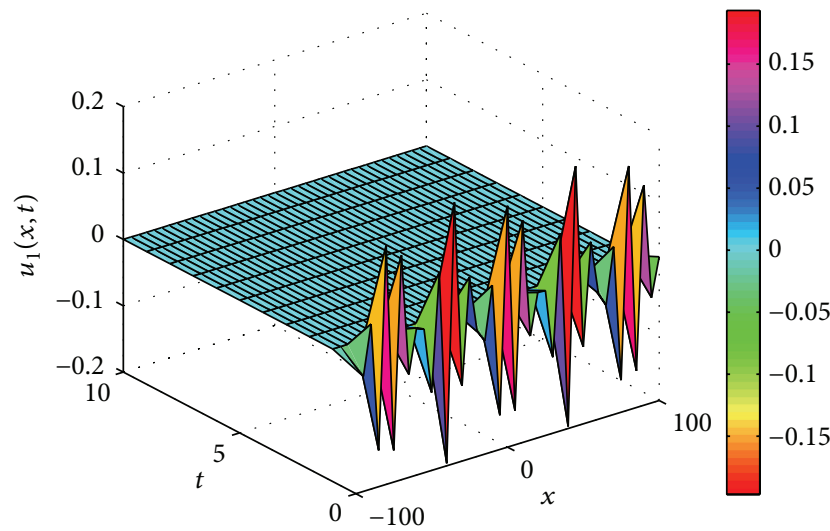

(c)

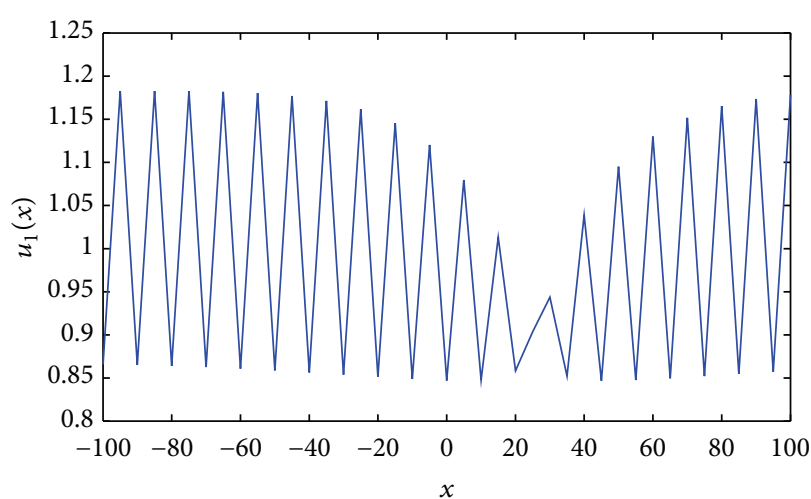

(b)

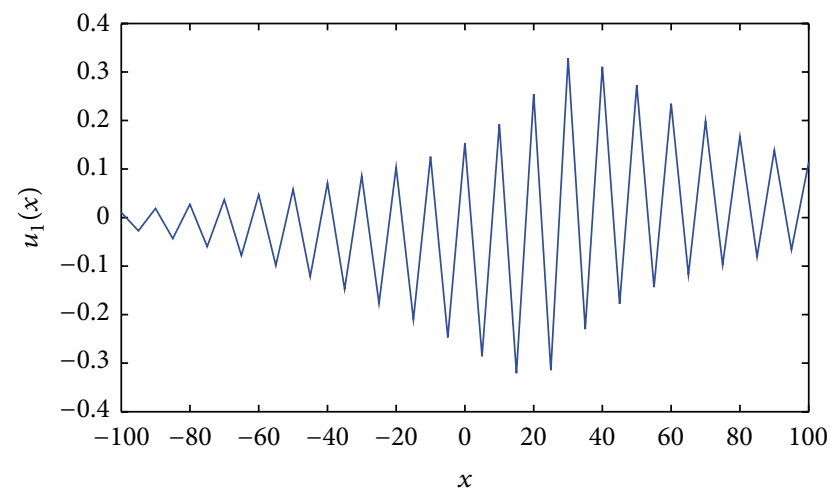

(d)

Figure 5: Graphs of ((a) and (b)) real values and ((c) and (d)) imaginary values of (14) are demonstrated at $C_{1}=2, p=2, \rho=2, a=$ 2, $\beta(t)=t+1, \gamma(t)=t-1$, and $\alpha(t)=t^{2}$ and by considering the values ((a) and (c)) $-100<x<100,0<t<10$ and ((b) and (d)) $-100<x<100, t=0.1$.

$$
\begin{gathered}
c(t)=\frac{1}{t} \int(\alpha(t) \\
\left.+(\rho+1) \sqrt{\frac{\gamma(t) \beta(t)}{2}}\right) d t+C_{1}, \\
u(\xi)=A_{0}+A_{1}\left[p+\tan \left(\frac{\Phi(\xi)}{2}\right)\right],
\end{gathered}
$$

where $c$ is an arbitrary constant. By using (114), Families 1, 2, and 14 can be written, respectively, as

$$
\begin{aligned}
& u_{89}(\xi)=1-p A_{1}+A_{1}\left[p+\frac{\rho-1}{2 A_{1}}\right. \\
& -\frac{\rho-1}{2 A_{1}} i
\end{aligned}
$$

$$
\begin{gathered}
\left.\cdot \tan \left(\frac{\sqrt{-c^{2}}(\rho-1)}{2 A_{1}}(\xi+C)\right)\right] \\
u_{90}(\xi)=1-p A_{1}+A_{1}\left[p+\frac{\rho-1}{2 A_{1}}\right. \\
\left.+\frac{\rho-1}{2 A_{1}} \tanh \left(\frac{\sqrt{c^{2}}(\rho-1)}{2 A_{1}}(\xi+C)\right)\right]
\end{gathered}
$$$$
u_{91}(\xi)=1-p A_{1}+A_{1}[p
$$$$
\left.-\frac{-\left(c(\rho-1) / A_{1}\right) e^{-\left(c(\rho-1) / A_{1}\right)(\xi+C)}}{c e^{-\left(c(\rho-1) / A_{1}\right)(\xi+C)}-1}\right] \text {, }
$$ 


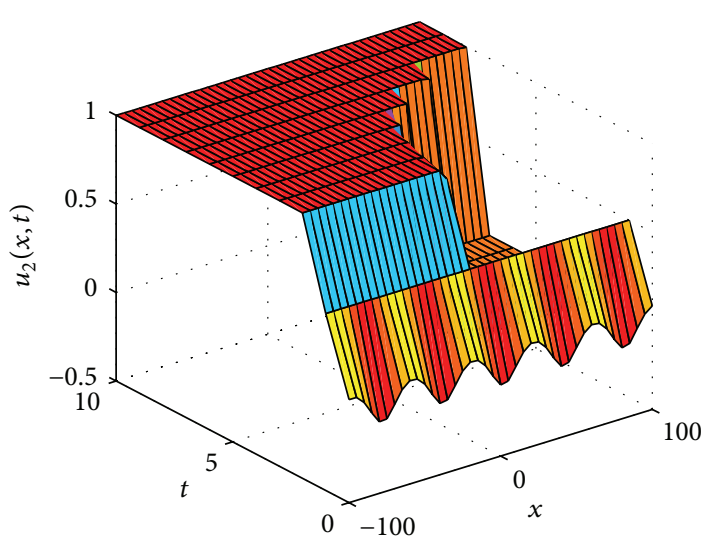

(a)

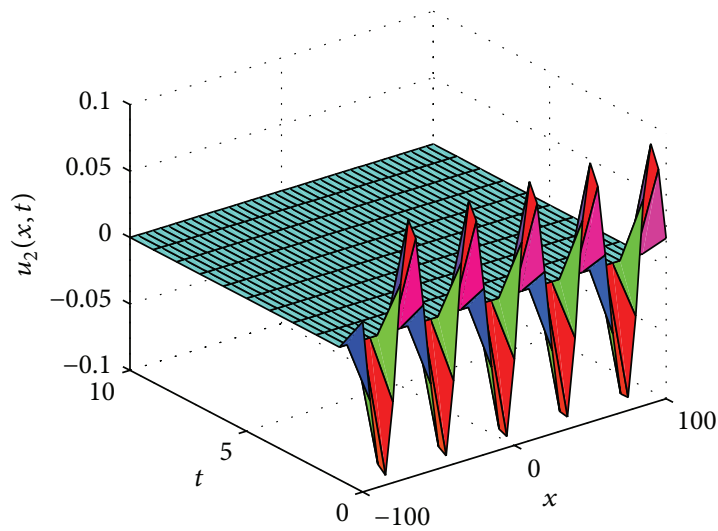

(c)

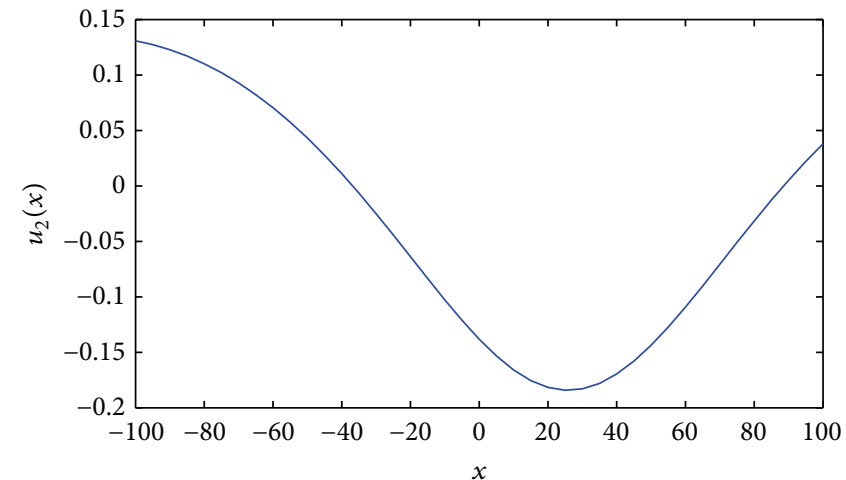

(b)

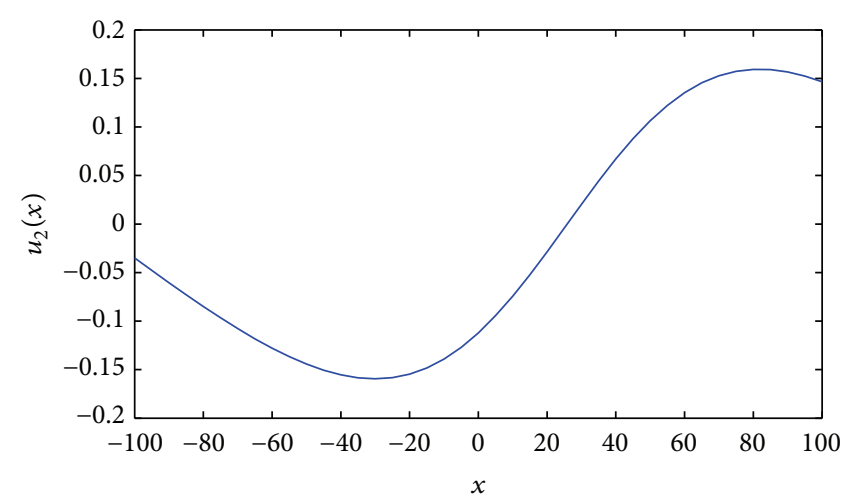

(d)

Figure 6: Graphs of ((a) and (b)) real values and ((c) and (d)) imaginary values of (15) are demonstrated at $C_{1}=2, p=2, k=2, \rho=$ 2, $a=2, \beta(t)=t+1, \gamma(t)=t-1$, and $\alpha(t)=t^{2}$ and by considering the values ((a) and (c)) $-100<x<100,0<t<10$ and ((b) and (d)) $-100<x<100, t=0.1$.

where $\xi=\left(A_{1} / c\right) \sqrt{\gamma(t) / 2 \beta(t)}\left(x-\int(\alpha(t)+(\rho+\right.$ 1) $\left.\sqrt{\gamma(t) \beta(t) / 2}) d t+C_{1}\right)$.

Set 39 . We have the following:

$$
\begin{aligned}
& \mu=\frac{A_{1}}{c} \sqrt{\frac{\gamma(t)}{2 \beta(t)}}, \\
& p=p, \\
& a=\frac{c}{A_{1}}, \\
& b=-c, \\
& c=c \\
& A_{0}=1-p A_{1}, \\
& A_{1}=A_{1}, \\
& B_{1}=0,
\end{aligned}
$$

$$
\begin{aligned}
& c(t)=\frac{1}{t} \int(\alpha(t) \\
& \left.-(2 \rho-1) \sqrt{\frac{\gamma(t) \beta(t)}{2}}\right) d t \\
& +C_{1}, \\
& u(\xi)=A_{0}+A_{1}\left[p+\tan \left(\frac{\Phi(\xi)}{2}\right)\right],
\end{aligned}
$$

where $c$ is an arbitrary constant. By using (117), Families 1, 2, and 14 can be written, respectively, as

$$
\begin{gathered}
u_{91}(\xi)=1-p A_{1}+A_{1}\left[p+\frac{1}{2 A_{1}}\right. \\
\left.-\frac{1}{2 A_{1}} i \tan \left(\frac{\sqrt{-c^{2}}}{2 A_{1}}(\xi+C)\right)\right],
\end{gathered}
$$




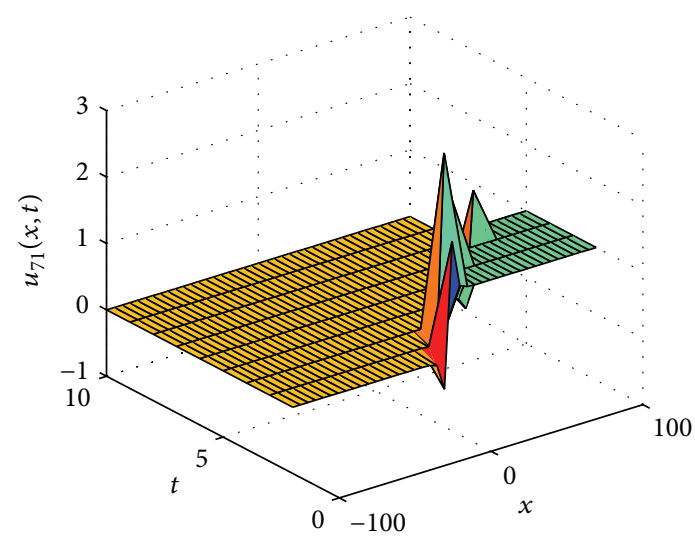

(a)

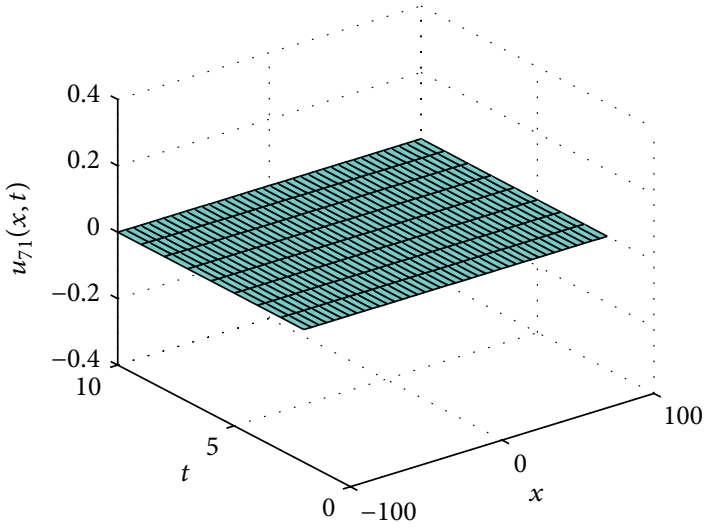

(c)
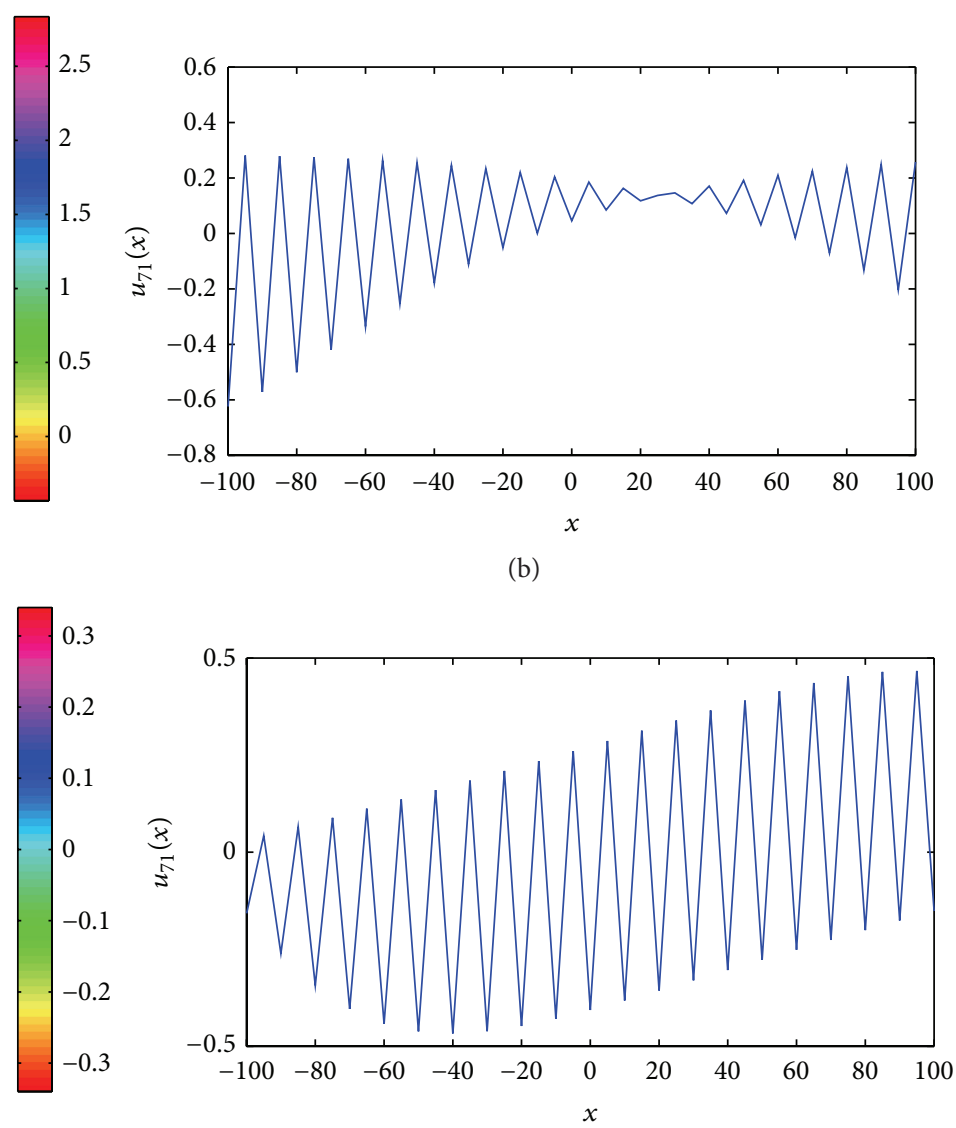

(d)

Figure 7: Graphs of ((a) and (b)) real values and ((c) and (d)) imaginary values of (96) are demonstrated at $C_{1}=2, p=2, k=2, \rho=$ 2, $c=2, \beta(t)=t+1, \gamma(t)=t-1$, and $\alpha(t)=t^{2}$ and by considering the values ((a) and (c)) $-100<x<100,0<t<10$ and ((b) and (d)) $-100<x<100, t=0.1$.

$$
\begin{aligned}
& u_{92}(\xi)=1-p A_{1}+A_{1}\left[p+\frac{1}{2 A_{1}}\right. \\
& \left.+\frac{1}{2 A_{1}} \tanh \left(\frac{\sqrt{c^{2}}}{2 A_{1}}(\xi+C)\right)\right], \\
& u_{93}(\xi)=1-p A_{1}+A_{1}[p \\
& \left.-\frac{\left(c / A_{1}\right) e^{\left(c / A_{1}\right)(\xi+C)}}{c e^{\left(c / A_{1}\right)(\xi+C)}-1}\right]
\end{aligned}
$$$$
\begin{aligned}
& a=-\frac{c \rho}{A_{1}}, \\
& b=-c, \\
& c=c, \\
& A_{0}=-p A_{1}, \\
& A_{1}=A_{1}, \\
& B_{1}=0, \\
& c(t)=\frac{1}{t} \int(\alpha(t)
\end{aligned}
$$

where $\xi=\left(A_{1} / c\right) \sqrt{\gamma(t) / 2 \beta(t)}\left(x-\int(\alpha(t)-(2 \rho-\right.$ 1) $\left.\sqrt{\gamma(t) \beta(t) / 2}) d t+C_{1}\right)$.

$$
\left.+(\rho-2) \sqrt{\frac{\gamma(t) \beta(t)}{2}}\right) d t
$$

Set 40 . We have the following:

$$
\begin{aligned}
& \mu=\frac{A_{1}}{c} \sqrt{\frac{\gamma(t)}{2 \beta(t)}}, \\
& p=p,
\end{aligned}
$$$$
+C_{1} \text {, }
$$$$
u(\xi)=A_{0}+A_{1}\left[p+\tan \left(\frac{\Phi(\xi)}{2}\right)\right]
$$ 


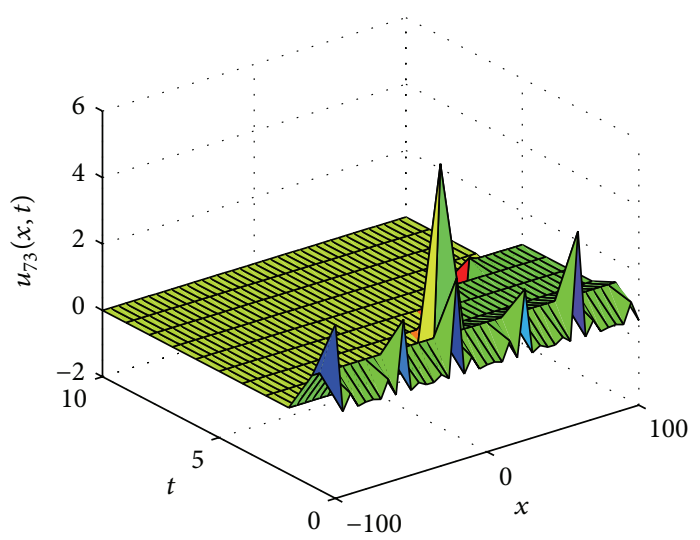

(a)

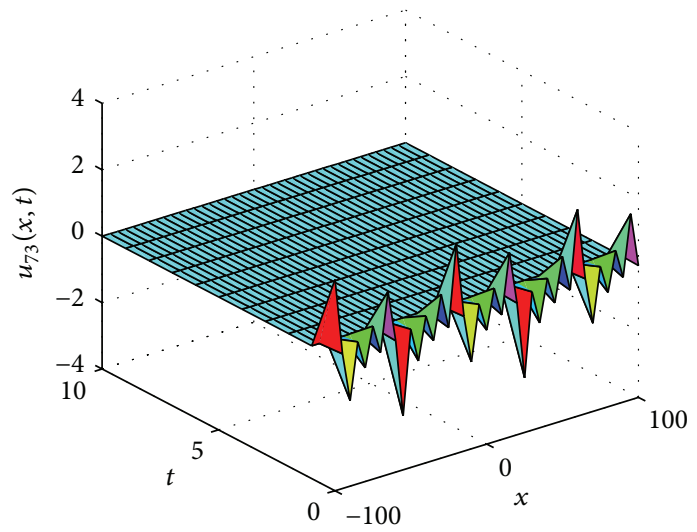

(c)

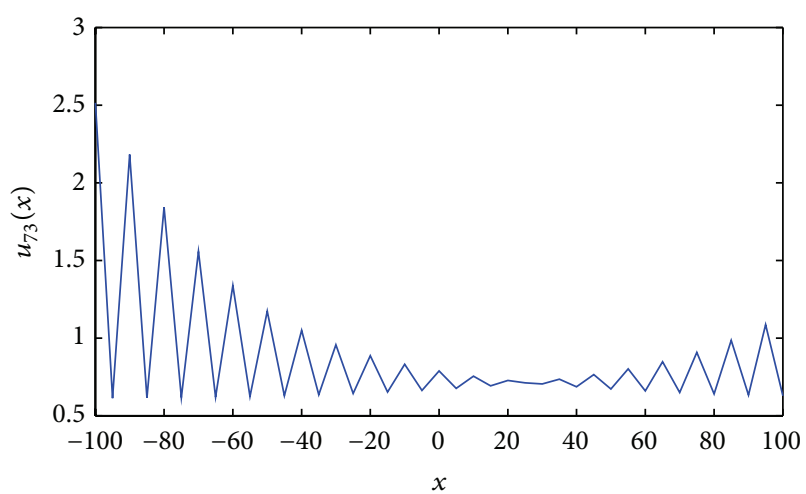

(b)

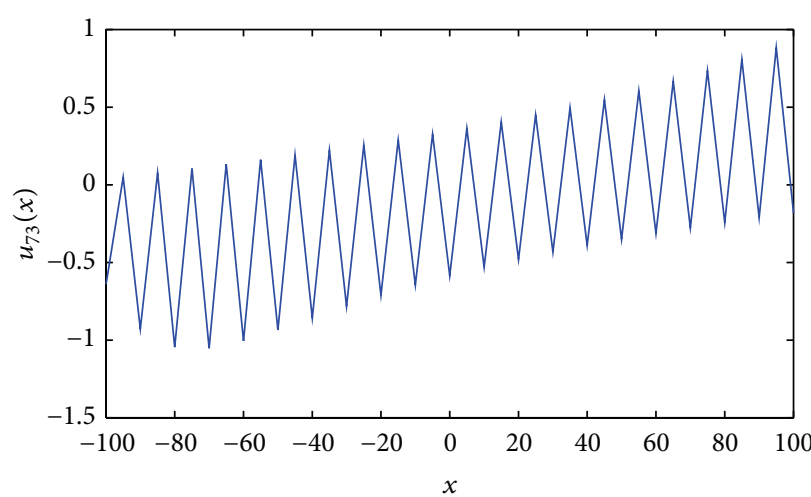

(d)

Figure 8: Graphs of ((a) and (b)) real values and ((c) and (d)) imaginary values of (97) are demonstrated at $C_{1}=2, p=2, k=2, \rho=$ 2, $c=2, \beta(t)=t+1, \gamma(t)=t-1$, and $\alpha(t)=t^{2}$ and by considering the values ((a) and (c)) $-100<x<100,0<t<10$ and ((b) and (d)) $-100<x<100, t=0.1$.

where $c$ is an arbitrary constant. By using (120), Families 1,2, and 14 can be written, respectively, as

$$
\begin{gathered}
u_{94}(\xi)=-p A_{1}+A_{1}\left[p+\frac{\rho}{2 A_{1}}\right. \\
-\frac{\rho}{2 A_{1}} i \tan \left(\frac{\sqrt{-c^{2}} \rho}{2 A_{1}}(\xi+C)\right), \\
u_{95}(\xi)=-p A_{1}+A_{1}\left[p+\frac{\rho}{2 A_{1}}\right. \\
\left.+\frac{\rho}{2 A_{1}} \tanh \left(\frac{\sqrt{c^{2}} \rho}{2 A_{1}}(\xi+C)\right)\right],
\end{gathered}
$$

$$
\begin{gathered}
u_{96}(\xi)=-p A_{1}+A_{1}[p \\
\left.-\frac{\left(c \rho / A_{1}\right) e^{\left(c \rho / A_{1}\right)(\xi+C)}}{c e^{\left(c \rho / A_{1}\right)(\xi+C)}-1}\right],
\end{gathered}
$$

where $\xi=\left(A_{1} / c\right) \sqrt{\gamma(t) / 2 \beta(t)}\left(x-\int(\alpha(t)+(\rho-\right.$ 2) $\left.\sqrt{\gamma(t) \beta(t) / 2}) d t+C_{1}\right)$.

Remark 1. In Figure 1, we plot two- and three-dimensional graphics of imaginary and real values of (14), which denote the dynamics of solutions with appropriate parametric selections. Also, in Figure 2, we draw two- and three-dimensional graphics of imaginary and real values of (15), which demonstrate the dynamics of solutions with convenient parametric choices. In Figure 3, we plot two- and three-dimensional graphics of (96), which represent the dynamics of solutions with proper parametric values. Moreover, in Figure 4, we 
draw two- and three-dimensional graphics of imaginary and real values of (97), which display the dynamics of solutions with suitable parametric selections. We plot two- and threedimensional graphics of Figures 1-4, when $-1000<x<$ 1000, $0<t<50$ and for Figures 5-8 when $-100<x<$ $100,0<t<10$. To the best of our knowledge, these complex exponential function solutions have not been submitted to the literature in advance. The analytical solutions and figures obtained in this paper give us a different physical interpretation for the nonlinear generalized Fitzhugh-Nagumo equation.

\section{Conclusion}

In this paper, the new approach of expansion method, namely, the improved $\tan (\Phi(\xi) / 2)$-expansion method, has successfully been implemented to investigate the generalized Fitzhugh-Nagumo equation with time-dependent coefficients. Abundant exact travelling wave solutions including solitons, kink, and periodic and rational solutions are attained. It is worth mentioning that some of the newly obtained solutions are identical to already published results. It has been shown that the applied method is effective and more wide ranging than the generalized and improved $\left(G^{\prime} / G\right)$ expansion method because it gives many new solutions. Therefore, this method can be applied to study many other nonlinear partial differential equations which frequently arise in engineering, mathematical physics, and other scientific real time application fields.

\section{Conflict of Interests}

The authors declare that there is no conflict of interests regarding the publication of this paper.

\section{References}

[1] P. Gray and S. K. Scott, Chemical Oscillations and InstabilitiesNon-Linear Chemical Kinetics, Oxford Science Publications, Oxford, UK, 1990.

[2] M. M. Kabir and A. Khajeh, "New explicit solutions for the Vakhnenko and a generalized form of the nonlinear heat conduction equations via exp-function method," International Journal of Nonlinear Sciences and Numerical Simulation, vol. 10, no. 10, pp. 1307-1318, 2009.

[3] M. Dehghan, J. Manafian, and A. Saadatmandi, "Solving nonlinear fractional partial differential equations using the homotopy analysis method," Numerical Methods for Partial Differential Equations, vol. 26, no. 2, pp. 448-479, 2010.

[4] M. G. Sakar and F. Erdogan, "The homotopy analysis method for solving the time-fractional Fornberg-Whitham equation and comparison with Adomian's decomposition method," Applied Mathematical Modelling, vol. 37, no. 20-21, pp. 88768885, 2013.

[5] M. G. Sakar and H. Ergören, "Alternative variational iteration method for solving the time-fractional Fornberg-Whitham equation," Applied Mathematical Modelling: Simulation and Computation for Engineering and Environmental Systems, vol. 39, no. 14, pp. 3972-3979, 2015.
[6] H. Jafari, A. Kadem, and D. Baleanu, "Variational iteration method for a fractional-order Brusselator system," Abstract and Applied Analysis, vol. 2014, Article ID 496323, 6 pages, 2014.

[7] M. Matinfar, S. Mirzanezhad, M. Ghasemi, and M. Salehi, "Solving the interaction of electromagnetic wave with electron by VIM," Journal of King Saud University-Science, vol. 27, no. 1, pp. 63-70, 2015.

[8] M. Dehghan and J. Manafian, "The solution of the variable coefficients fourth-order parabolic partial differential equations by the homotopy perturbation method," Zeitschrift für Naturforschung A, vol. 64, no. 7-8, pp. 420-430, 2009.

[9] E. Cuce and P. M. Cuce, "A successful application of homotopy perturbation method for efficiency and effectiveness assessment of longitudinal porous fins," Energy Conversion and Management, vol. 93, pp. 92-99, 2015.

[10] A.-M. Wazwaz, "Travelling wave solutions for combined and double combined sine-cosine-Gordon equations by the variable separated ODE method," Applied Mathematics and Computation, vol. 177, no. 2, pp. 755-760, 2006.

[11] J. Manafian Heris and M. Lakestani, "Solitary wave and periodic wave solutions for variants of the KdV-Burger and the $\mathrm{K}(\mathrm{n}$, n)-Burger equations by the generalized tanh-coth method," Communications in Numerical Analysis, vol. 2013, 18 pages, 2013.

[12] J. Manafian Heris and M. Lakestani, "Exact solutions for the integrable sixth-order drinfeld-sokolov-satsuma-hirota system by the analytical methods," International Scholarly Research Notices, vol. 2014, Article ID 840689, 8 pages, 2014.

[13] J. Manafian and I. Zamanpour, "Exact travelling wave solutions of the symmetric regularized long wave (SRLW) using analytical methods," Statistics, Optimization \& Information Computing, vol. 2, no. 1, pp. 47-55, 2014.

[14] M. A. Abdou and A. A. Soliman, "Modified extended tanhfunction method and its application on nonlinear physical equations," Physics Letters A, vol. 353, no. 6, pp. 487-492, 2006.

[15] S. A. El-Wakil and M. A. Abdou, "New exact travelling wave solutions using modified extended tanh-function method," Chaos, Solitons \& Fractals, vol. 31, no. 4, pp. 840-852, 2007.

[16] J. Manafian Heris and M. Bagheri, "Exact solutions for the modified KDV and the generalized KDV equations via Expfunction method," Journal of Mathematical Extension, vol. 4, no. 2, pp. 77-98, 2010.

[17] J. Manafian Heris and I. Zamanpour, "Analytical treatment of the coupled Higgs equation and the Maccari system via Expfunction method," Acta Universitatis Apulensis, vol. 33, pp. 203216, 2013.

[18] M. G. Hafez, M. N. Alam, and M. A. Akbar, "Traveling wave solutions for some important coupled nonlinear physical models via the coupled Higgs equation and the Maccari system," Journal of King Saud University - Science, vol. 27, no. 2, pp. 105$112,2015$.

[19] H.-O. Roshid and M. Azizur Rahman, "The $\exp (-\phi(\eta))$ expansion method with application in the $(1+1)$-dimensional classical Boussinesq equations," Results in Physics, vol. 4, pp. 150-155, 2014.

[20] M. Fazli Aghdaei and J. Manafianheris, "Exact solutions of the couple Boiti-Leon-Pempinelli system by the generalized $\left(G^{\prime} / G\right)$-expansion method," Journal of Mathematical Extension, vol. 5, no. 2, pp. 91-104, 2011.

[21] H. Naher and F. A. Abdullah, "New approach of $\left(G^{\prime} / G\right)$ expansion method and new approach of generalized $\left(G^{\prime} / G\right)$ expansion method for nonlinear evolution equation," AIP Advances, vol. 3, Article ID 032116, 2013. 
[22] A. J. M. Jawad, M. D. Petkovic, and A. Biswas, "Modified simple equation method for nonlinear evolution equations," Applied Mathematics and Computation, vol. 217, no. 2, pp. 869-877, 2010.

[23] M. N. Alam, M. A. Akbar, and S. T. Mohyud-Din, "A novel $\left(G^{\prime} / G\right)$-expansion method and its application to the Boussinesq equation," Chinese Physics B, vol. 23, no. 2, Article ID 020203, 2014.

[24] Y. Chen and Q. Wang, "Extended Jacobi elliptic function rational expansion method and abundant families of Jacobi elliptic function solutions to $(1+1)$-dimensional dispersive long wave equation," Chaos, Solitons and Fractals, vol. 24, no. 3, pp. 745-757, 2005.

[25] X. Zhao, L. Wang, and W. Sun, “The repeated homogeneous balance method and its applications to nonlinear partial differential equations," Chaos, Solitons \& Fractals, vol. 28, no. 2, pp. 448-453, 2006

[26] A. Hajipour and S. M. Mahmoudi, "Application of exp-function method to Fitzhugh-Nagumo equation," World Applied Sciences Journal, vol. 9, no. 1, pp. 113-117, 2010.

[27] J. A. Whitehead and A. C. Newell, "Finite bandwidth, finite amplitude convection," Journal of Fluid Mechanics, vol. 38, no. 2, pp. 279-303, 1969.

[28] A. H. Bhrawy, "A Jacobi-Gauss-Lobatto collocation method for solving generalized Fitzhugh-Nagumo equation with timedependent coefficients," Applied Mathematics and Computation, vol. 222, pp. 255-264, 2013.

[29] M. Dehghan, J. M. Heris, and A. Saadatmandi, "Application of semi-analytic methods for the Fitzhugh-Nagumo equation, which models the transmission of nerve impulses," Mathematical Methods in the Applied Sciences, vol. 33, no. 11, pp. 1384-1398, 2010.

[30] R. FitzHugh, "Impulses and physiological states in theoretical models of nerve membrane," Biophysical Journal, vol. 1, no. 6, pp. 445-466, 1961.

[31] J. Nagumo, S. Arimoto, and S. Yoshizawa, "An active pulse transmission line simulating nerve axon," Proceedings of the IRE, vol. 50, no. 10, pp. 2061-2070, 1962.

[32] G. Hariharan and K. Kannan, "Haar wavelet method for solving FitzHugh-Nagumo equation," World Academy of Science, Engineering and Technology, vol. 67, pp. 560-564, 2010.

[33] T. Kawahara and M. Tanaka, "Interactions of traveling fronts: an exact solution of a nonlinear diffusion equation," Physics Letters A, vol. 97, no. 8, pp. 311-314, 1983.

[34] M. C. Nucci and P. A. Clarkson, "The nonclassical method is more general than the direct method for symmetry reductions: an example of the FitzHugh-Nagumo equation," Physics Letters $A$, vol. 164, no. 1, pp. 49-56, 1992.

[35] H. Li and Y. Guo, "New exact solutions to the FitzHughNagumo equation," Applied Mathematics and Computation, vol. 180, no. 2, pp. 524-528, 2006.

[36] H. Triki and A.-M. Wazwaz, "On soliton solutions for the Fitzhugh-Nagumo equation with time-dependent coefficients," Applied Mathematical Modelling, vol. 37, no. 6, pp. 3821-3828, 2013.

[37] R. Jiwari, R. K. Gupta, and V. Kumar, "Polynomial differential quadrature method for numerical solutions of the generalized Fitzhugh-Nagumo equation with time-dependent coefficients," Ain Shams Engineering Journal, vol. 5, no. 4, pp. 1343-1350, 2014.

[38] Q. Zheng and J. Shen, "Pattern formation in the FitzHughNagumo model," Computers \& Mathematics with Applications, vol. 70, no. 5, pp. 1082-1097, 2015.
[39] R. A. Van Gorder, "Gaussian waves in the Fitzhugh-Nagumo equation demonstrate one role of the auxiliary function $H(x$, $t$ ) in the homotopy analysis method," Communications in Nonlinear Science and Numerical Simulation, vol. 17, no. 3, pp. 1233-1240, 2012.

[40] F. Liu, P. Zhuang, I. Turner, V. Anh, and K. Burrage, "A semialternating direction method for a 2-D fractional FitzHughNagumo monodomain model on an approximate irregular domain," Journal of Computational Physics, vol. 293, pp. 252263, 2015.

[41] A. H. Abbasian, H. Fallah, and M. R. Razvan, "Symmetric bursting behaviors in the generalized FitzHugh-Nagumo model," Biological Cybernetics, vol. 107, no. 4, pp. 465-476, 2013.

[42] S. S. Ray and S. Sahooz, "New exact solutions of fractional zakharov-kuznetsov and modified zakharov-kuznetsov equations using fractional sub-equation method," Communications in Theoretical Physics, vol. 63, no. 1, pp. 25-30, 2015.

[43] S. Sahoo and S. S. Ray, "Improved fractional sub-equation method for $(3+1)$-dimensional generalized fractional KdVZakharov-Kuznetsov equations," Computers \& Mathematics with Applications, vol. 70, no. 2, pp. 158-166, 2015. 


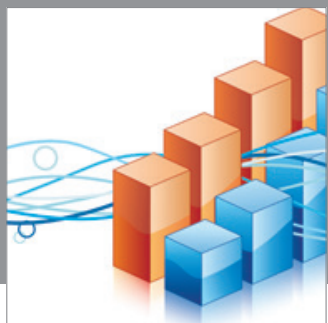

Advances in

Operations Research

mansans

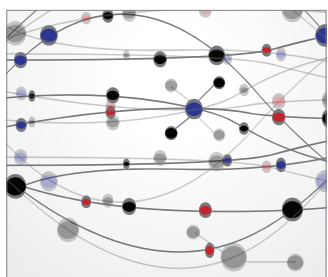

The Scientific World Journal
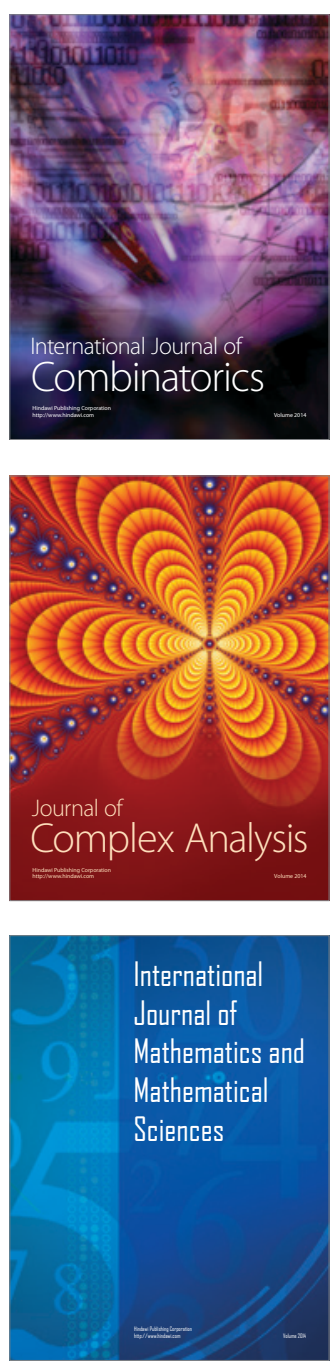
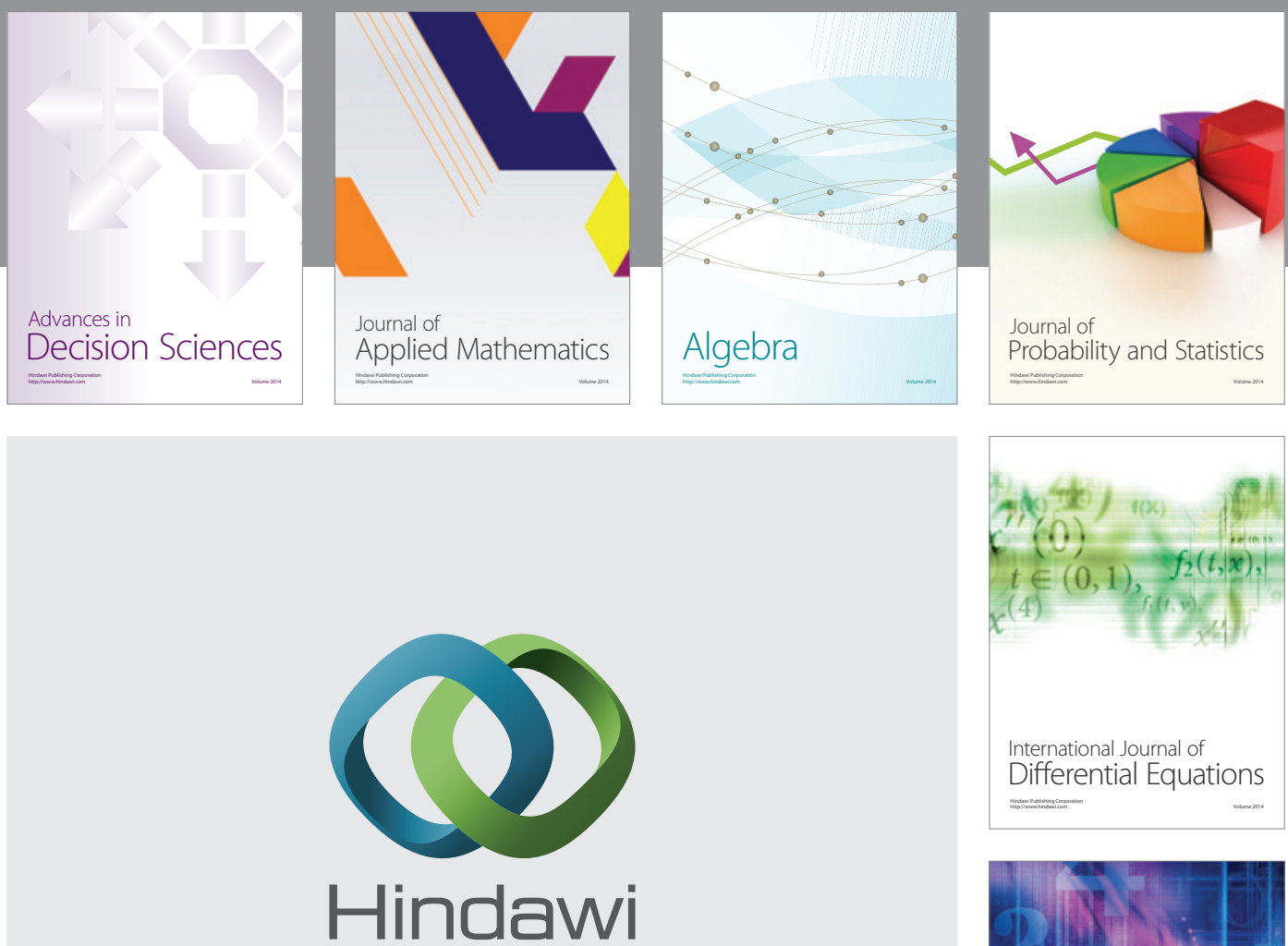

Submit your manuscripts at http://www.hindawi.com
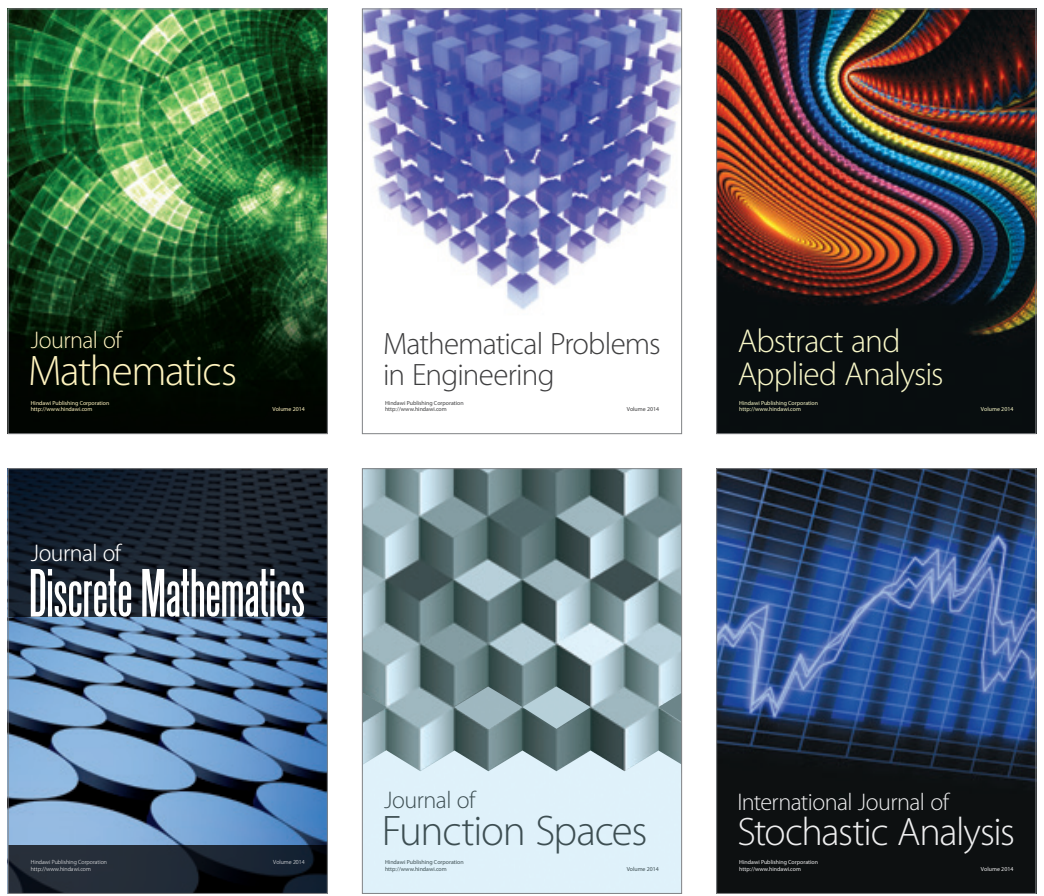

Journal of

Function Spaces

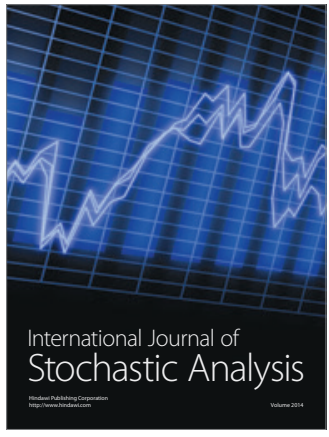

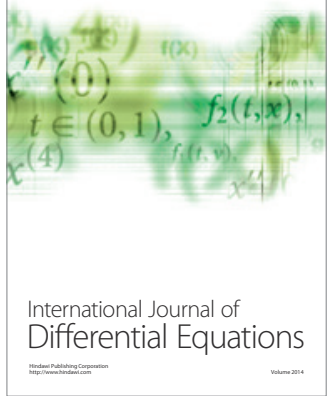
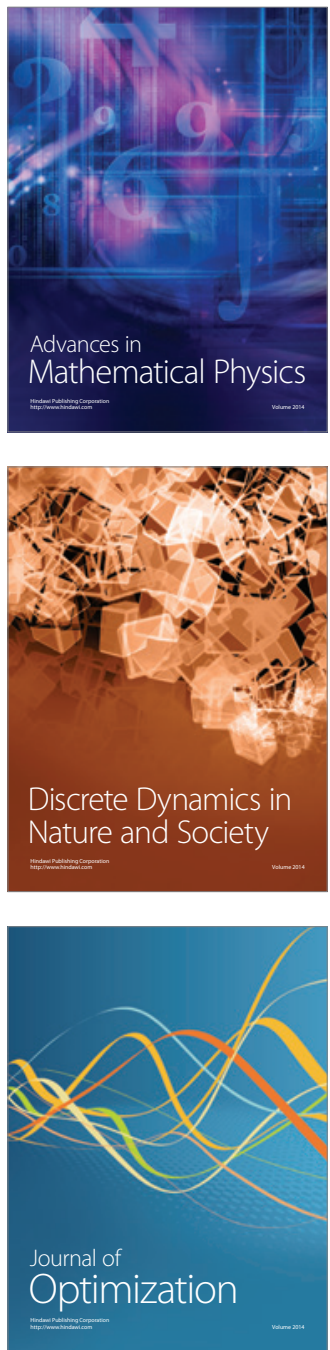